\title{
An ethnopharmacological survey and comparative analysis of plants from the Sudhnoti District, Azad Jammu and Kashmir, Pakistan
}

Muhammad Faraz Khan ${ }^{1}$, Zia-ur-Rahman Mashwani ${ }^{2}$, Ansar Mehmood ${ }^{1}$, Rehmatullah Qureshi', Rizwan Sarwar ${ }^{2}$, Khawaja Shafique Ahmad ${ }^{1}$ and Cassandra L. Quave ${ }^{3^{*}}$ (1)

\begin{abstract}
Background: This is the first comprehensive report on the traditional and novel uses of medicinal plants practiced by the indigenous communities of the Sudhnoti district of Azad Jammu and Kashmir (AJK), Pakistan. The area is rich in folklore and indigenous medicinal knowledge due to a unique tribal composition and socioeconomic conditions. This study aimed to document traditional knowledge of native plant use by the local communities, particularly those used for therapeutic purposes.

Methods: Field surveys were conducted from September 2015 to March 2017. Interviews with 125 local inhabitants of different tribes, age groups, genders, and occupations were conducted using structured and semi-structured questions along with group discussions. Data gathered on plant uses, local names, and modes of application of each plant species were organized in tables. Ethnobotanical indices such as use value (UV) and cultural significance index (CSI) were used to produce quantitative information on the plant use category, frequency, and cultural preference of species. Reports on therapeutic uses of medicinal plants were compared with previous studies.

Results: In all, 88 plant species from 45 families were reported, out of which 67 (77\%) were used in ethnomedical applications. Asteraceae, Rosaceae, Fabaceae, and Lamiaceae were the dominant families. Berberis lycium was the most valued plant species, followed by Zanthoxylum armatum and Taraxacum officinale. Mentha arvensis had the highest cultural significance, followed by Mentha longifolia, Punica granatum, and Zanthoxylum armatum. Leaves were the most preferred plant parts in the preparation of medicine exclusively or mixed with other parts. The most frequently used process of crude preparation of medicinal plants was cooking. Oral intake was the predominant route of administration.

(Continued on next page)
\end{abstract}

\footnotetext{
* Correspondence: cquave@emory.edu

${ }^{3}$ Center for the Study of Human Health, Emory University, 550 Asbury Circle, Atlanta, GA 30322, USA

Full list of author information is available at the end of the article
}

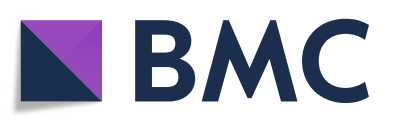

(- The Author(s). 2021 Open Access This article is licensed under a Creative Commons Attribution 4.0 International License, which permits use, sharing, adaptation, distribution and reproduction in any medium or format, as long as you give appropriate credit to the original author(s) and the source, provide a link to the Creative Commons licence, and indicate if changes were made. The images or other third party material in this article are included in the article's Creative Commons licence, unless indicated otherwise in a credit line to the material. If material is not included in the article's Creative Commons licence and your intended use is not permitted by statutory regulation or exceeds the permitted use, you will need to obtain permission directly from the copyright holder. To view a copy of this licence, visit http://creativecommons.org/licenses/by/4.0/. The Creative Commons Public Domain Dedication waiver (http://creativecommons.org/publicdomain/zero/1.0/) applies to the data made available in this article, unless otherwise stated in a credit line to the data. 
(Continued from previous page)

Conclusions: Our comparative analysis confirmed that most of the plants documented have uses that match those previously reported for the region and other parts of the world, with the exception of novel medicinal uses for 11 plant species, including Verbascum thapsus for earache, Elaeagnus umbellata for hepatitis, Achillea millefolium for oral care, Dicliptera roxburghiana to prevent sunstroke in cattle, Rumex hastatus for allergy antidote, Pyrus pashia for hepatitis, and Nerium oleander for diabetes.

Keywords: Medicinal plants, Ethnomedicine, Pakistan, Sudhnoti

\section{Background}

Azad Jammu and Kashmir (AJ\&K) is a predominantly Himalayan state in the north of Pakistan located $90 \mathrm{~km}$ away from Islamabad, Pakistan's capital city. The northwestern border of the district is pierced by the Jhelum River from the Rawalpindi district of Pakistan. The area is hilly, striped by narrow valleys along the course of nullahs and streams, nesting a dense rural population of approximately 300,000 people, according to the 2017 census. Studies have shown that the Himalayan region is home to more than 10,000 medicinal and aromatic plants, and local communities primarily rely on herbal medicine to meet their primary healthcare needs [1]. Other studies revealed that more than $80 \%$ of the Pakistani population is reliant solely or primarily on traditional medicine for their everyday health and livelihood [2].

Owing to a unique tribal composition, where up to $85 \%$ of the district population belongs to the "Sudhan" tribe, the Sudhnoti district has conserved extensive traditional folklore and practices. On the other hand, discrete communities of other ethnic groups, especially the Gujjars, have a legacy of plant uses ranging from food to medicine [3]. The dependence of local communities on traditional medicine can also be linked to poor healthcare infrastructure in the area. The local socioeconomic conditions are characterized by low per capita income compared to other parts of the country, leading to an overwhelming dependence on biodiversity for sustenance and medicine.

Plants are vital for human survival as a source of food, fuel, fodder, timber, and medicine [4]. Recent studies have confirmed large amounts of pollen in fossil spectra from Georgia dating back to the Upper Paleolithic Period [5], indicating the prehistoric relationship of plants with humankind. Ethnobotanical studies aim to document the folk uses of medicinal plants by local communities to understand the traditional interdependence of plants and people. Current approaches in ethnobotany include the scientific study of plant-people relationships for the latter's well-being in a sustainable manner. The use of relative cultural indices is an emerging trend in ethnobotany, which provides data amenable to hypothesis testing and comparative analysis [6]. Additionally, primary data reported with comprehensive use descriptions and comparative accounts can help rationalize any possible complexity arising from indices [7]. According to a study conducted in the Sudhnoti District, local communities of the area are highly dependent on plants for their therapeutic uses; therefore, documentation of their medicinal knowledge, as well as pharmacological validation thereof, is highly encouraged [8].

Although ethnobotanical studies have been conducted on a large scale in the surrounding areas of AJK [9-12], ethnomedicinal knowledge in the Sudhnoti District remains poorly studied. To the best of our knowledge, a single attempt was made that focused primarily on medicinal plants without acknowledging novel therapeutic uses of medicinal plants [9]. The aim of our study was two-fold: (1) to document the ethnobotanical knowledge of the area and (2) to compare it with other studies conducted in the surrounding regions and other parts of the world at large in efforts to identify novel medicinal plant uses that could merit further pharmacological evaluation.

\section{Methods}

\section{Study area}

The Sudhnoti District, AJ\&K, study area lies in coordinates ranging from $33^{\circ} .40^{\prime}-33^{\circ} 50^{\prime} \mathrm{N}$ latitude and 73.40 $73^{\circ} 50^{\prime} \mathrm{E}$ longitude. The altitude range is approximately 600 to 2100 m.a.s.l. (Fig. 1). The study area is comprised of hills and mountains along with small valleys and plains, spanning a total area of about $5695 \mathrm{~km}^{2}$. During the summer and winter seasons, the temperature ranges between 20 to $35^{\circ} \mathrm{C}$ and $5{ }^{\circ} \mathrm{C}$ and $20^{\circ} \mathrm{C}$, respectively.

\section{Ethnographic composition}

The study area has a distinct tribal composition with a majority of the population belonging to Sudhan tribe, followed by Awan, Syed, Gujjar, Qureshi, and Kashmiri ethnic groups. Nearly, the entire population lives in a rural setting with a strong religious influence. Hence, gender discrepancies still exist in the health and education sectors and the acquisition of traditional knowledge. When researchers approach a typical rural household, the elder male is most likely the source of traditional knowledge, especially when the researcher is male. In small towns, including the district headquarters, there is an appreciable number of educated youths possessing 


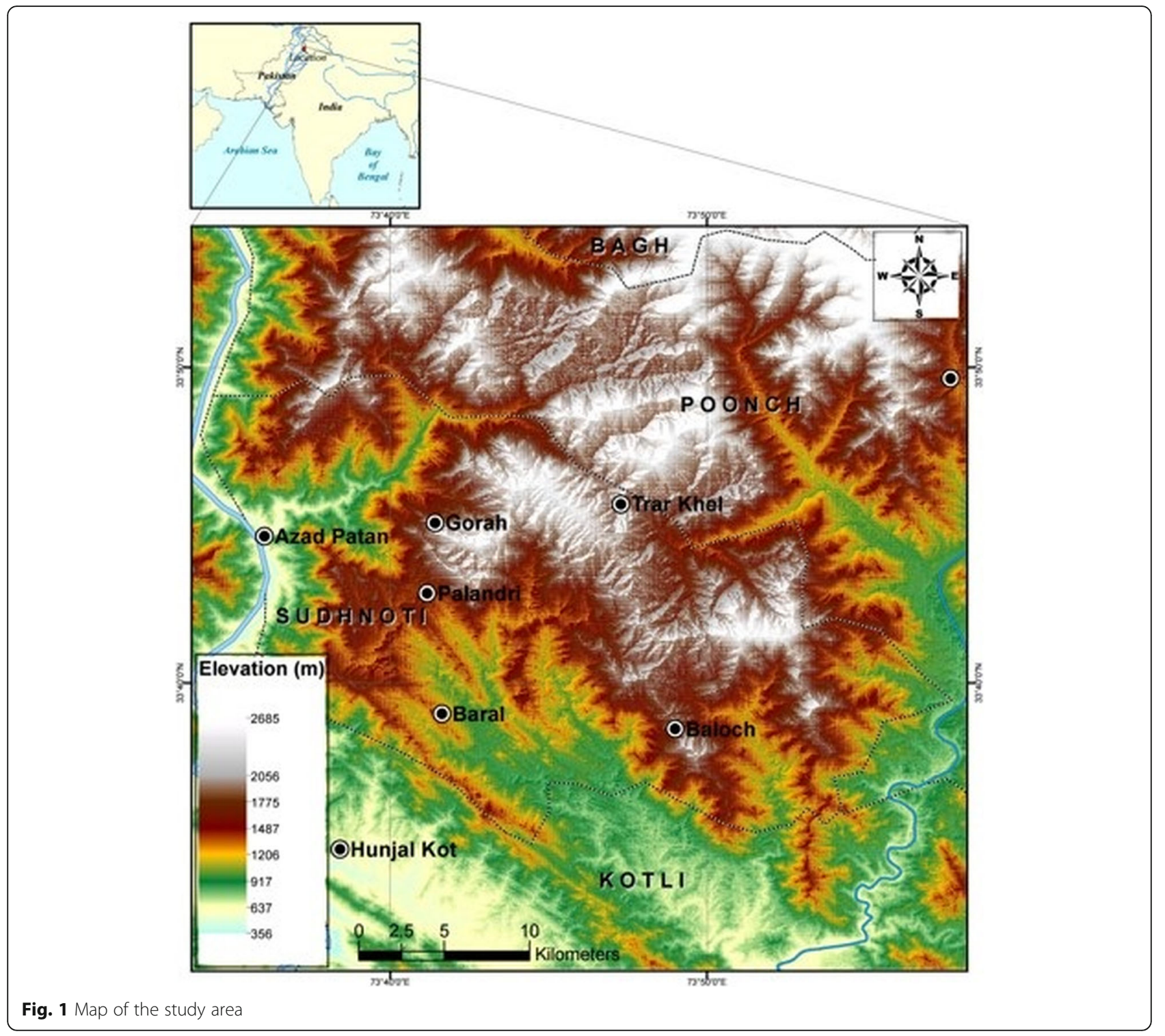

common knowledge of plant uses. However, the bulk of traditional folklore is still conserved with the illiterate elderly adults, living in the remote highlands and riverside pastures. Besides small-scale conventional farming and livestock rearing, most of the population survives directly on biodiversity and work on daily wages for sustenance. Pahari is the most commonly spoken language in the area, with different dialects spoken. Only a few informants from the Gujjar tribe prefer to speak Gojri while discussing traditional uses of plants; the same is true for elderly women from the Kashmiri tribe. The conventional healthcare infrastructure comprises a district headquarter hospital with a dozen doctors and less than ten basic health units in the entire district. On average, there is one physician per 7000 citizens in the district. Access to physicians is even more restricted for the people living in remote areas as there is a very underdeveloped road network in the study area.

\section{Sampling and data collection}

Six different sites in the Sudhnoti District were selected based on altitude and demographic composition for sampling and data collection, namely, Azad Pattan, Baral, Baloch, Palandri, Gorah, and Trarkhal (Fig. 1). Prior to data collection, a preliminary survey was conducted in which local administration and community stakeholders were engaged for prior consent. Multiple field surveys were conducted from September 2015 to March 2017. Prior informed consent was always verbally obtained before conducting interviews, and the ethical standards of the Society for Economic Botany and International Society of Ethnobiology were followed [13]. Open-ended interviews and structured questions followed field surveys and transect walks either at common places where local people gather (e.g., local bazaars) or at the residences of informants. The first author 
conducted all interviews in the local language (Pahari) and subsequently documented the interviews in Urdu and English. Local people with biomedical and geographical knowledge were also involved whenever required for better communication, such as understanding disease symptoms. Local healers known as "Pansari," and nomadic healers locally known as "Sunyasi," were interviewed with temporarily mounted plant samples.

During fieldwork, 88 plant specimens were collected, preserved, identified, and indexed. A total of 121 informants of 30-85 years old were interviewed (Table 1). The gender division of infomants was 55\% male and $45 \%$ women. Collected plant specimens were dried, pressed, and mounted on herbarium sheets. The identification of plants was carried out with the assistance of Dr. Rehmatullah Qureshi at PMAS Arid Agriculture University. The Flora of Pakistan [14] was used as a standard for identification and nomenclature. Voucher specimens were submitted to the herbarium of PMAS Arid Agriculture University, and accession numbers were acquired. Botanical nomenclature followed the most updated accepted names included (http://www. theplantlist.org) and family designations followed the Angiosperm Phylogeny Group IV assignments [15]. Digital images of the collected herbarium specimens are available in Additional file 1.

\section{Use value (UV)}

Use value, a primary measure of all reported uses of a plant species cited by an informant was calculated by the following formula

$$
\mathrm{UV}=\sum \mathrm{U}_{\mathrm{i}} / \mathrm{N}
$$

where $U_{\mathrm{i}}$ is the number of uses revealed by each informant for a given species and $N$ is the total number of informants.

\section{Cultural significance index (CSI)}

CSI is an anthropological index developed by [16] and modified by [17]. CSI calculates the importance of plant taxon through a researcher-generated weight ranking of multiple factors. A two-point scale for the variables such as use frequency $(2=$ species effectively used, $1=$ not the preferred use), management, and preference were used. To reduce the sensitivity of the method to sampling intensity, a correction factor is also incorporated as in the consensus method [17, 18].

$$
\mathrm{CSI}=\Sigma(\mathrm{i} \times \mathrm{e} \times \mathrm{c}) \times \mathrm{CF}
$$

Here, " $i$ " refers to the species management, " $e$ " is the preference of use of a plant species by informant for a specific purpose, and " $c$ " is the frequency of use reported.

\begin{tabular}{|c|c|c|c|}
\hline Variables & Categories & No of informants & Percentage \\
\hline \multirow[t]{2}{*}{ Gender of informants } & Male & 66 & 54.77 \\
\hline & Female & 55 & 45.23 \\
\hline \multirow[t]{3}{*}{ Age of informants } & 25 to 45 & 26 & 20.60 \\
\hline & 46 to 65 & 49 & 39.66 \\
\hline & Above 65 & 46 & 37.19 \\
\hline \multirow[t]{3}{*}{ Marital status } & Married & 95 & 78.51 \\
\hline & Unmarried & 21 & 17.35 \\
\hline & Widow & 5 & 4.13 \\
\hline \multirow[t]{4}{*}{ Education status } & Illiterate & 59 & 48.76 \\
\hline & Secondary school & 23 & 19.00 \\
\hline & College & 26 & 21.48 \\
\hline & University & 13 & 10.74 \\
\hline \multirow[t]{3}{*}{ Employment status } & Farmer & 27 & 22.31 \\
\hline & Shepherd & 34 & 28.09 \\
\hline & Other & 60 & 49.58 \\
\hline \multirow[t]{3}{*}{ Ethnic group } & Sudhan & 82 & 67.76 \\
\hline & Gujar & 19 & 15.70 \\
\hline & Others & 20 & 30.37 \\
\hline \multirow[t]{3}{*}{ Language } & Pahari & 114 & 94.21 \\
\hline & Gojri & 4 & 3.30 \\
\hline & Kashmiri & 3 & 2.47 \\
\hline
\end{tabular}

Table 1 The demographic composition of the informants 


\section{Comparative analysis}

Primary ethnobotanical uses of all plant species were compared to regional studies containing "ethnobotany," "medicinal plants," "folklore," or related search terms using different databases [19]. Dissimilar uses cited by other researchers were reviewed and cited. To avoid repetition of similar uses reported in multiple articles for a particular species, the one chosen for citation was the one conducted nearest to the immediate study region.

\section{Novelty assessment}

A total of 13 plant species were identified as being reported for novel uses from the region. These plant species were subjected to a robust comparative review using literature databases, i.e., PubMed, Scifinder, and NAPR ALERT, to develop a broader picture of their previously reported pharmacological activities and cross-examining the novelty of uses reported in the present study. Novel uses are highlighted in bold in Table 2 .

\section{Results}

\section{Diversity of medicinal plants}

During the first phase of this study, 88 plant species from 47 families were tabulated after taxonomic identification and scrutiny. Asteraceae was the dominant family with eight plant species, followed by Rosaceae (6), Fabaceae (6), and Lamiaceae (5). Other significant families were Poaceae and Acanthaceae, each with four plant species (Fig. 2). All other plant species represented 45 angiosperm families. The data show that angiosperms contribute to $97 \%$ of the collected plant species, followed by ferns (3\%).

\section{Medicinal taxa cited by different ethnic groups}

The informants belonging to different ethnic origins were grouped into three major categories: Sudhan, Gujjar, and Others (Kashmiri, Syed, Quereshit, etc.). Sudhans, owing to their largest share in population, cited the highest number of plants (48), Gujjars cited 21, and others reported 16. There was also an overlap of taxa between ethnic groups: six between Sudhan and Gujjars and five between Sudhan and Others. Only four plants were commonly cited by all three groups (Fig. 3).

\section{Ethnobotanical uses and therapeutic value}

Out of the total 88 plant species, $78 \%$ of plants were used for therapeutic purposes, followed by food (18\%), and wood (8\%). Plant species having aesthetic or religious value are grouped together (Fig. 4). The used categories are highly overlapping, as different plant parts of certain species were reported for different uses by different community sections simultaneously.

\section{Parts used for medicinal purposes}

Among the plant species used for medicinal purposes, leaves were the most commonly used plants parts (37\%), followed by aerial parts (23\%), and fruits (18\%). However, in the case of medicinal herbs, the use of aerial parts and the whole plant is dominant (Fig. 5). The use of bark and roots is culturally discouraged due to the local tendency toward resource conservation.

\section{Method of preparation of folk recipes}

The largest share of plant species $(20 \%)$ had a reported use as cooked vegetables. However, the share of plants used as infusions (21\%) and decoctions/teas (13\%) together was $34 \%$ of all the species (Fig. 6). Interestingly, there is a notable trend of multi-plant formulations devised by semiprofessional herbalists and traditional practitioners. In these cases, the powder of more than one plant or plant part is orally administered with water in a formula known as "Phakki." There are some instances with such recipes where overdose and malpractice resulted in adverse drug reactions [66].

\section{Route of administration}

With the ancient mindset that human ailments result from an abnormality in the body's internal system, most of the botanical preparations are taken orally (85\%). External application is restricted to pain management, bone fractures, wounds, and skin infections. A couple of plants were reported for nasal inhalation and as drops for the eyes or ears. The use of certain plant species as chewing sticks (Zanthoxylum armatum DC, Rutaceae, and Nerium oleander L., Apocynaceae) is also considered internal here for ease of classification.

\section{Use value}

A total of 20 plant species were identified as the most valuable in the area (Table 2). Berberis lyceum, with a use-value of 0.343 , was the most valued plant species followed by Zanthoxylum armatum (0.28), Taraxacum officinale (0.26), Solanum villosum (0.26), Swertia chirata (0.21), and Mentha longifolia (0.1), cited mostly for their medicinal use-value. Dodonaea viscoa (0.23), Pyrus pashia (0.19), and Ficus palmata (0.18) were reported with moderately high use-values.

\section{CS}

Cultural significance of a plant species is markedly affected by preference, management, and frequency of use by local inhabitants. Mentha arvensis, with a value of 9.9, has the highest cultural significance followed by Mentha longifolia (9.7), Berberis lyceum (9.6), Justicia adhatoda (8.8), and Zanthxylum armatum (6.7). Other species with cultural significance are Punica granatum, Artemisia absinthium, and Swertia chiraita (Table 2). 
Table 2 Ethnobotanical profile of plants from the area. Plants with novel medicinal uses are highlighted in bold

\begin{tabular}{|c|c|c|c|c|c|c|c|c|c|c|c|}
\hline Family & BN. Acc. & LN & PU & $\operatorname{Rec}$ & App & Uses & $N$ & UV & CSI & $\begin{array}{l}\text { Previously } \\
\text { reported uses }\end{array}$ & References \\
\hline \multirow[t]{4}{*}{ Acanthaceae } & $\begin{array}{l}\text { Dicliptera } \\
\text { roxburghiana } \\
\text { Nees. FK-5006 }\end{array}$ & & Aerial parts & Fodder & Oral & $\begin{array}{l}\text { Used to treat } \\
\text { sun stroke in } \\
\text { buffaloes. }\end{array}$ & 1 & 0.023 & 1.70 & $\begin{array}{l}\text { The powder is } \\
\text { used as a } \\
\text { general tonic }\end{array}$ & {$[20]$} \\
\hline & $\begin{array}{l}\text { Justicia } \\
\text { adhatoda L. FK- } \\
5022\end{array}$ & Bhekar & Leaves & Wrap & Topical & $\begin{array}{l}\text { Used with } \\
\text { onion, } \\
\text { turmeric and } \\
\text { oil for papules } \\
\text { and pimples. }\end{array}$ & 3 & 0.103 & 8.86 & $\begin{array}{l}\text { Used for } \\
\text { wound } \\
\text { infections. } \\
\text { Decoction is } \\
\text { antispasmodic, } \\
\text { expectorant } \\
\text { and } \\
\text { abortifacient }\end{array}$ & {$[21]$} \\
\hline & $\begin{array}{l}\text { Nerium } \\
\text { oleander L. FK- } \\
5038\end{array}$ & Kneera & $\begin{array}{l}\text { Shoots/ } \\
\text { flowers }\end{array}$ & $\begin{array}{l}\text { Powder, } \\
\text { aerial } \\
\text { parts }\end{array}$ & Oral & $\begin{array}{l}\text { Used to treat } \\
\text { diabetes. } \\
\text { Used to treat } \\
\text { gum } \\
\text { bleeding and } \\
\text { toothache. }\end{array}$ & 7 & 0.139 & 7.25 & $\begin{array}{l}\text { Used to treat } \\
\text { stomach pain. } \\
\text { Used to treat } \\
\text { ear pain and } \\
\text { eyes problems. } \\
\text { Considered } \\
\text { poisonous. }\end{array}$ & [22] \\
\hline & $\begin{array}{l}\text { Strobilanthes } \\
\text { attenuata Nees } \\
\text { FK- } 5065\end{array}$ & Malol & Flower & & & $\begin{array}{l}\text { Used to } \\
\text { prepare local } \\
\text { dyes. }\end{array}$ & 1 & 0.007 & 2.40 & $\begin{array}{l}\text { The decoction } \\
\text { is used for the } \\
\text { treatment of } \\
\text { boils. It is also } \\
\text { laxative and } \\
\text { anti-helminthic. }\end{array}$ & [23] \\
\hline Amaranthaceae & $\begin{array}{l}\text { Amaranthus } \\
\text { spinosus L. FK- } \\
4986\end{array}$ & Ganar & Aerial parts & Vegetable & Oral & $\begin{array}{l}\text { Cooked as } \\
\text { vegetable } \\
\text { Effective } \\
\text { against } \\
\text { constipation } \\
\text { and obesity. } \\
\text { Used as } \\
\text { fodder. }\end{array}$ & 8 & 0.103 & 2.40 & $\begin{array}{l}\text { Seeds are } \\
\text { cooked with } \\
\text { rice and given } \\
\text { for joint pain. }\end{array}$ & [24] \\
\hline Apocynaceae & $\begin{array}{l}\text { Carissa spinarum } \\
\text { L. FK-4995 }\end{array}$ & Granda & Fruit & & & Fruit is edible & 2 & 0.021 & 3.00 & $\begin{array}{l}\text { Fruit is used as } \\
\text { a blood purifier. }\end{array}$ & {$[25]$} \\
\hline Araliaceae & $\begin{array}{l}\text { Hedera helix L. } \\
\text { FK-5019 }\end{array}$ & Batkal & Leaves & Infusion & Oral & $\begin{array}{l}\text { Used to treat } \\
\text { diabetes. }\end{array}$ & 6 & 0.029 & 1.60 & $\begin{array}{l}\text { Used for } \\
\text { benign warts }\end{array}$ & [26] \\
\hline \multirow[t]{4}{*}{ Asteraceae } & $\begin{array}{l}\text { Achillea } \\
\text { millefolium } \\
\text { L.FK-4984 }\end{array}$ & Kangi & Root & Infusion & Oral & $\begin{array}{l}\text { Root extract } \\
\text { is used to } \\
\text { treat gum } \\
\text { problems. } \\
\text { Infusion is } \\
\text { used to } \\
\text { remove } \\
\text { kidney } \\
\text { stone. }\end{array}$ & 4 & 0.022 & 3.60 & $\begin{array}{l}\text { Used for fever, } \\
\text { colds, and } \\
\text { urinary } \\
\text { disorders. Aerial } \\
\text { parts are used } \\
\text { to improve bile } \\
\text { flow. }\end{array}$ & {$[27]$} \\
\hline & $\begin{array}{l}\text { Artemisia } \\
\text { vulgaris L. FK- } \\
4988\end{array}$ & Chaoo & Aerial parts & Vegetable & Oral & $\begin{array}{l}\text { Cooked as } \\
\text { vegetables. } \\
\text { Decoction is } \\
\text { used for } \\
\text { fevers. }\end{array}$ & 6 & 0.044 & 2.00 & $\begin{array}{l}\text { Kills parasitic } \\
\text { worms. Leaf } \\
\text { extract is used } \\
\text { for malaria and } \\
\text { fever. }\end{array}$ & [28] \\
\hline & $\begin{array}{l}\text { Artemisia } \\
\text { absinthium L. } \\
\text { FK-4989 }\end{array}$ & Isanteen & Whole plant & Powder & Oral & $\begin{array}{l}\text { Dried ground } \\
\text { powder of } \\
\text { whole plant is } \\
\text { used as } \\
\text { hypoglycemic. }\end{array}$ & 8 & 0.120 & 4.00 & $\begin{array}{l}\text { Leaf powder is } \\
\text { used for gastric } \\
\text { issues. Paste is } \\
\text { applied on } \\
\text { teeth for pain } \\
\text { relief }\end{array}$ & {$[28]$} \\
\hline & $\begin{array}{l}\text { Cichorium } \\
\text { intybus L. FK- } \\
4998\end{array}$ & Kasni & Leaves & $\begin{array}{l}\text { Vegetable/ } \\
\text { decoction }\end{array}$ & Oral & $\begin{array}{l}\text { Cooked as a } \\
\text { vegetable; } \\
\text { decoction is } \\
\text { used to treat } \\
\text { liver } \\
\text { inflammation. }\end{array}$ & & 0.059 & 6.00 & $\begin{array}{l}\text { Leaves are } \\
\text { used as } \\
\text { vegetables. } \\
\text { Roots and } \\
\text { leaves are used } \\
\text { as a diuretic, }\end{array}$ & [29] \\
\hline
\end{tabular}


Table 2 Ethnobotanical profile of plants from the area. Plants with novel medicinal uses are highlighted in bold (Continued)

\begin{tabular}{|c|c|c|c|c|c|c|c|c|c|c|c|}
\hline Family & BN. Acc. & LN & PU & $\operatorname{Rec}$ & App & Uses & $N$ & UV & CSI & $\begin{array}{l}\text { Previously } \\
\text { reported uses }\end{array}$ & References \\
\hline & & & & & & $\begin{array}{l}\text { Laxative, } \\
\text { effective } \\
\text { against } \\
\text { constipation. }\end{array}$ & & & & $\begin{array}{l}\text { laxative and } \\
\text { tonic. Used for } \\
\text { fever, gas } \\
\text { trouble, and } \\
\text { body swelling. } \\
\text { Effective for } \\
\text { stomach } \\
\text { problems. }\end{array}$ & \\
\hline & $\begin{array}{l}\text { Conyza } \\
\text { canadensis (L.) } \\
\text { Cronquist. FK- } \\
5000\end{array}$ & Gandi booti & & & & $\begin{array}{l}\text { Reported as } \\
\text { allergic. } \\
\text { Causes skin } \\
\text { irritations. }\end{array}$ & 1 & 0.01 & 0.24 & Uses as fodder & [10] \\
\hline & $\begin{array}{l}\text { Oplismenus } \\
\text { compositus (L.) } \\
\text { P. P.Beauv. FK- } \\
5041\end{array}$ & Chura kaha & Aerial parts & & & Fodder grass & 4 & 1.290 & 1.37 & Fodder grass & [22] \\
\hline & $\begin{array}{l}\text { Taraxacum } \\
\text { officinale F.H. } \\
\text { Wigg. FK- } 5071\end{array}$ & Hand & Leaves & Vegetable & Oral & $\begin{array}{l}\text { Cooked as } \\
\text { vegetable for } \\
\text { women after } \\
\text { child birth. } \\
\text { Used as } \\
\text { antidiabetic } \\
\text { and for } \\
\text { jaundice. }\end{array}$ & 6 & 0.260 & 8.00 & $\begin{array}{l}\text { Used to treat } \\
\text { bone fractures. } \\
\text { Used for cattle } \\
\text { after delivery } \\
\text { for } 15 \text { days for } \\
\text { strengthening } \\
\text { bones and } \\
\text { muscles. }\end{array}$ & [30] \\
\hline & $\begin{array}{l}\text { Tagetes minuta } \\
\text { L. FK- } 5072\end{array}$ & Satbarga & Inflorescence & Ointment & Topical & $\begin{array}{l}\text { Used as } \\
\text { ointments for } \\
\text { skin care by } \\
\text { females. }\end{array}$ & 4 & 0.135 & 2.60 & $\begin{array}{l}\text { Used for skin } \\
\text { disease. } \\
\text { Effective } \\
\text { against fungal } \\
\text { infections. Used } \\
\text { in fevers and } \\
\text { fits, for piles } \\
\text { and earache. }\end{array}$ & [31] \\
\hline Berberendiaceae & $\begin{array}{l}\text { Berberis lycium } \\
\text { Royle FK-4992 }\end{array}$ & Sumblo & Root bark & Decoction & Oral & $\begin{array}{l}\text { Decoction of } \\
\text { root bark is } \\
\text { boiled and } \\
\text { used for } \\
\text { wound } \\
\text { healing and } \\
\text { as anti- } \\
\text { infective. }\end{array}$ & 10 & 0.163 & 9.60 & $\begin{array}{l}\text { Used to treat } \\
\text { external injuries } \\
\text { and to stop } \\
\text { bleeding. }\end{array}$ & [32] \\
\hline \multirow[t]{2}{*}{ Boraginaceae } & $\begin{array}{l}\text { Cynoglossum } \\
\text { lanceolatum } \\
\text { Forssk. FK-5003 }\end{array}$ & & & Infusion & Oral & $\begin{array}{l}\text { Aphrodisiac, } \\
\text { demulcent }\end{array}$ & 4 & 0.140 & 3.41 & $\begin{array}{l}\text { Infantile } \\
\text { diarrhea, heals } \\
\text { sores, wounds, } \\
\text { joins cracked } \\
\text { and fractured } \\
\text { bones and } \\
\text { relieves swollen } \\
\text { limbs }\end{array}$ & [33] \\
\hline & $\begin{array}{l}\text { Trichodesma } \\
\text { indicum (L.) } \\
\text { Lehm. FK- } 5075\end{array}$ & & Roots & & Oral & $\begin{array}{l}\text { Used for } \\
\text { inflammation }\end{array}$ & 2 & 1.331 & 1.87 & $\begin{array}{l}\text { Ethno- } \\
\text { veterinary uses }\end{array}$ & [34] \\
\hline \multirow[t]{2}{*}{ Brassicaceae } & $\begin{array}{l}\text { Capsella bursa- } \\
\text { pastoris (L.) } \\
\text { Medik. FK-4994 }\end{array}$ & Trepatri & Whole plant & Infusion & Oral & $\begin{array}{l}\text { Whole plant is } \\
\text { used for } \\
\text { intestinal } \\
\text { problems, i.e. } \\
\text { vomiting. } \\
\text { Used as } \\
\text { fodder. }\end{array}$ & 3 & 0.014 & 3.00 & $\begin{array}{l}\text { Decocotion of } \\
\text { the plant is } \\
\text { used to lower } \\
\text { blood pressure } \\
\text { and abdominal } \\
\text { pain, bile } \\
\text { secretion, } \\
\text { obesity and } \\
\text { hemorrhages. }\end{array}$ & [35] \\
\hline & $\begin{array}{l}\text { Sorghum } \\
\text { halepense FK- }\end{array}$ & & Leaves & Vegetable & Oral & $\begin{array}{l}\text { Cooked as } \\
\text { vegetable for }\end{array}$ & 2 & 0.014 & 6.60 & $\begin{array}{l}\text { Rhizome is } \\
\text { used by }\end{array}$ & [27] \\
\hline
\end{tabular}


Table 2 Ethnobotanical profile of plants from the area. Plants with novel medicinal uses are highlighted in bold (Continued)

\begin{tabular}{|c|c|c|c|c|c|c|c|c|c|c|c|}
\hline Family & BN. Acc. & LN & PU & $\operatorname{Rec}$ & App & Uses & $N$ & UV & CSI & $\begin{array}{l}\text { Previously } \\
\text { reported uses }\end{array}$ & References \\
\hline & 5037 & & & & & $\begin{array}{l}\text { its } \\
\text { carminative } \\
\text { effect. }\end{array}$ & & & & $\begin{array}{l}\text { "hakeems" for } \\
\text { stomach pain } \\
\text { and indigestion }\end{array}$ & \\
\hline Buxaceae & $\begin{array}{l}\text { Sarcococca } \\
\text { saligna (D.Don) } \\
\text { Müll. Arg. FK- } \\
5059\end{array}$ & Naidroon & Leaves & Infusion & Oral & $\begin{array}{l}\text { Root extract } \\
\text { is used to } \\
\text { treat } \\
\text { toothache. } \\
\text { Leaves are } \\
\text { antipyretic. } \\
\text { Used for } \\
\text { thatching }\end{array}$ & 5 & 0.022 & 5.0 & $\begin{array}{l}\text { Used for } \\
\text { hypertension, } \\
\text { hypoglycemia. }\end{array}$ & {$[36]$} \\
\hline Convolvulaceae & $\begin{array}{l}\text { Convolvulus } \\
\text { arvensis L. FK- } \\
4997\end{array}$ & & Aerial parts & Vegetable & Oral & $\begin{array}{l}\text { Used as } \\
\text { fodder. }\end{array}$ & 4 & 0.088 & 1.80 & & \\
\hline Cypraceae & $\begin{array}{l}\text { Eriophorum } \\
\text { comosum (Wall.) } \\
\text { Nees FK-5012 }\end{array}$ & Smakh & Aerial parts & & & Fodder grass & 8 & 0.074 & 0.93 & $\begin{array}{l}\text { Used for } \\
\text { abdominal pain } \\
\text { care. }\end{array}$ & {$[37]$} \\
\hline Dryopteridaceae & $\begin{array}{l}\text { Dryopteris } \\
\text { ramose (C. } \\
\text { Hope) C. Chr. } \\
\text { FK-5008 }\end{array}$ & Leengri & Aerial parts & vegetable & Oral & $\begin{array}{l}\text { Used as a } \\
\text { vegetable to } \\
\text { treat ulcer } \\
\text { and } \\
\text { constipation. }\end{array}$ & 6 & 0.037 & 1.70 & $\begin{array}{l}\text { Plant juice is } \\
\text { used for } \\
\text { stomach pain. } \\
\text { Fronds are } \\
\text { used as fodder } \\
\text { for horses. }\end{array}$ & {$[38]$} \\
\hline Ebenaceae & $\begin{array}{l}\text { Diospyros lotus } \\
\text { L. FK-5007 }\end{array}$ & Amlook & Fruit/ leaves & Food & Oral & $\begin{array}{l}\text { Fruit eaten to } \\
\text { cure stomach } \\
\text { troubles, } \\
\text { leaves used as } \\
\text { fodder and } \\
\text { wood for fuel. }\end{array}$ & 4 & 0.111 & 4.80 & $\begin{array}{l}\text { Juice of unripe } \\
\text { fruit in used to } \\
\text { lower blood } \\
\text { pressure. Fruit } \\
\text { is used as a } \\
\text { remedy for } \\
\text { hiccups. }\end{array}$ & {$[20,38]$} \\
\hline Eleagnaceae & $\begin{array}{l}\text { Elaeagnus } \\
\text { umbellata } \\
\text { Thunb. FK- } \\
5009\end{array}$ & Kankooli & Fruit & Juice & Oral & $\begin{array}{l}\text { Fruit is edible } \\
\text { and fruit juice } \\
\text { is taken as a } \\
\text { treatment for } \\
\text { chronic liver } \\
\text { problems } \\
\text { including } \\
\text { hepatitis. }\end{array}$ & 7 & 0.177 & 9.10 & $\begin{array}{l}\text { Root is edible. } \\
\text { Green parts are } \\
\text { used as a } \\
\text { source of } \\
\text { forage. Wood is } \\
\text { used for fuel. }\end{array}$ & {$[20,39]$} \\
\hline \multirow[t]{2}{*}{ Euphorbiaceae } & $\begin{array}{l}\text { Euphorbia } \\
\text { helioscopia L. } \\
\text { FK-5011 }\end{array}$ & Dodhal & Aerial parts & Latex & Oral & $\begin{array}{l}\text { Used to treat } \\
\text { constipation. }\end{array}$ & 7 & 0.070 & 3.20 & $\begin{array}{l}\text { Roots are used } \\
\text { as anthelmintic. } \\
\text { Aerial parts are } \\
\text { used for } \\
\text { constipation. } \\
\text { Seeds are used } \\
\text { for cholera. }\end{array}$ & [10] \\
\hline & $\begin{array}{l}\text { Mallotus } \\
\text { philippensis } \\
\text { (Lam.) Mill Arg. } \\
\text { FK-5029 }\end{array}$ & Kamella & Fruit & & & $\begin{array}{l}\text { Considered } \\
\text { toxic due to } \\
\text { its purgative } \\
\text { effects. Fruit } \\
\text { was used to } \\
\text { extract red } \\
\text { dye } \\
\text { previously. }\end{array}$ & 2 & 0.092 & 1.80 & $\begin{array}{l}\text { Anthelminthic } \\
\text { effects are } \\
\text { reported }\end{array}$ & [40] \\
\hline \multirow[t]{2}{*}{ Fabaceae } & $\begin{array}{l}\text { Astragalus } \\
\text { psilacanthus } \\
\text { Boiss. FK-4990 }\end{array}$ & Kanda & Aerial parts & & & $\begin{array}{l}\text { Used as } \\
\text { fodder. }\end{array}$ & 5 & 0.031 & 1.13 & $\begin{array}{l}\text { Leaves are } \\
\text { used for flu } \\
\text { and toothache. }\end{array}$ & {$[28]$} \\
\hline & $\begin{array}{l}\text { Indigofera } \\
\text { heterantha Wall } \\
\text { Brandis FK-5020 }\end{array}$ & Jand & Aerial parts & Infusion & & $\begin{array}{l}\text { Used for } \\
\text { stomach } \\
\text { disorders. } \\
\text { Used to make } \\
\text { baskets. Used } \\
\text { as fodder. }\end{array}$ & 3 & 0.074 & 2.50 & . & \\
\hline
\end{tabular}


Table 2 Ethnobotanical profile of plants from the area. Plants with novel medicinal uses are highlighted in bold (Continued)

\begin{tabular}{|c|c|c|c|c|c|c|c|c|c|c|c|}
\hline Family & BN. Acc. & $\mathrm{LN}$ & PU & $\operatorname{Rec}$ & App & Uses & $N$ & UV & CSI & $\begin{array}{l}\text { Previously } \\
\text { reported uses }\end{array}$ & References \\
\hline & $\begin{array}{l}\text { Lespedeza juncea } \\
\text { Wall FK-5023 }\end{array}$ & Jandi & Aerial parts & & & Fodder grass & 4 & 0.170 & 0.79 & $\begin{array}{l}\text { Used to treat a } \\
\text { variety of skin } \\
\text { diseases }\end{array}$ & [9] \\
\hline & $\begin{array}{l}\text { Lotus } \\
\text { corniculatus L. } \\
\text { FK-5024 }\end{array}$ & Trepatri & Aerial parts & & & Fodder & 5 & 0.195 & 0.69 & & \\
\hline & $\begin{array}{l}\text { Melilotus indica } \\
\text { L. All. FK-5033 }\end{array}$ & Sree kaha & Leaves & Vegetable & Oral & $\begin{array}{l}\text { Cooked as } \\
\text { vegetable. } \\
\text { Seeds are } \\
\text { used to treat } \\
\text { genital } \\
\text { infection }\end{array}$ & 1 & 0.07 & 2.0 & $\begin{array}{l}\text { Infusion is used } \\
\text { in cough and } \\
\text { bronchial } \\
\text { disorders. }\end{array}$ & [3] \\
\hline & $\begin{array}{l}\text { Trifolium repens } \\
\text { L. FK- } 5074\end{array}$ & Shatahl & Leaves & Vegetable & Oral & $\begin{array}{l}\text { Used to treat } \\
\text { gout. Used as } \\
\text { fodder. }\end{array}$ & 3 & 0.037 & 3.48 & $\begin{array}{l}\text { Anti-rheumatic } \\
\text { and depurative, } \\
\text { used in cough, } \\
\text { cold, gout and } \\
\text { leucorrhoea. }\end{array}$ & [41] \\
\hline Fagaceae & $\begin{array}{l}\text { Quercus incana } \\
\text { W. Bartram. FK- } \\
5050\end{array}$ & Erruti & Seeds & Powder & Oral & $\begin{array}{l}\text { A major } \\
\text { source of fuel } \\
\text { wood. Seeds } \\
\text { are used to } \\
\text { treat } \\
\text { dysentery. } \\
\text { Used to make } \\
\text { traditional } \\
\text { plough. }\end{array}$ & 3 & 0.109 & 1.30 & $\begin{array}{l}\text { Astringent, } \\
\text { used in } \\
\text { diarrhea and } \\
\text { indigestion, } \\
\text { wood is used } \\
\text { to make } \\
\text { agriculture } \\
\text { Tools. }\end{array}$ & {$[12,38]$} \\
\hline \multirow[t]{3}{*}{ Gentianaceae } & $\begin{array}{l}\text { Gentiana } \\
\text { olivieri (Griseb) } \\
\text { FK-5017 }\end{array}$ & Neel kanth & Leaves & Decoction & Oral & $\begin{array}{l}\text { Decoction is } \\
\text { used for } \\
\text { cough. Roots } \\
\text { are used to } \\
\text { treat } \\
\text { infection of } \\
\text { genitals in } \\
\text { male child. }\end{array}$ & 6 & 0.051 & 7.80 & $\begin{array}{l}\text { Used for } \\
\text { cardiac } \\
\text { ailments. Root } \\
\text { is used for } \\
\text { stomachache }\end{array}$ & {$[42]$} \\
\hline & $\begin{array}{l}\text { Swertia chirata } \\
\text { Buch.-Ham. ex } \\
\text { Wall. FK- } 5066\end{array}$ & Chraita & Leaves & Infusion & Oral & $\begin{array}{l}\text { Used against } \\
\text { fevers i.e. } \\
\text { typhoid and } \\
\text { malarial } \\
\text { fevers. }\end{array}$ & 3 & 0.213 & 5.40 & $\begin{array}{l}\text { The decoction } \\
\text { is used for the } \\
\text { treatment of } \\
\text { boils. Laxative } \\
\text { and anti- } \\
\text { helminthic }\end{array}$ & [10] \\
\hline & $\begin{array}{l}\text { Swertia alata } \\
\text { C.B.Clarke. FK- } \\
5067\end{array}$ & Chraita & Whole plant & Decoction & Oral & $\begin{array}{l}\text { Used for fever } \\
\text { and liver } \\
\text { inflammation }\end{array}$ & 4 & 0.201 & 3.33 & & \\
\hline \multirow[t]{2}{*}{ Geraniaceae } & $\begin{array}{l}\text { Geranium } \\
\text { wallichianum D. } \\
\text { Don ex Sweet } \\
\text { FK-5015 }\end{array}$ & & Leaves & Paste & Topical & $\begin{array}{l}\text { Leaf paste is } \\
\text { used for joint } \\
\text { pain. }\end{array}$ & 4 & 0.044 & 1.60 & $\begin{array}{l}\text { Root extract is } \\
\text { used in chronic } \\
\text { diarrhea. } \\
\text { Rhizome is } \\
\text { used in powder } \\
\text { form for } \\
\text { backache, } \\
\text { mouth ulcers } \\
\text { and chronic } \\
\text { diarrhea }\end{array}$ & [43] \\
\hline & $\begin{array}{l}\text { Geranium } \\
\text { nepalense Sweet } \\
\text { FK-5016 }\end{array}$ & & Roots & Tea & Oral & $\begin{array}{l}\text { Gives relief } \\
\text { from pains, } \\
\text { i.e. migraine. } \\
\text { Used to treat } \\
\text { renal } \\
\text { disorders. }\end{array}$ & 7 & 0.022 & 3.50 & $\begin{array}{l}\text { Astringent, } \\
\text { used to cure } \\
\text { renal problems. }\end{array}$ & [44] \\
\hline Juglandaceae & $\begin{array}{l}\text { Juglans regia L. } \\
\text { FK- } 5021\end{array}$ & Khour & Bark & $\begin{array}{l}\text { Chewing } \\
\text { stick }\end{array}$ & Oral & $\begin{array}{l}\text { Bark is used } \\
\text { as chewing } \\
\text { sticks to cure }\end{array}$ & 4 & 0.150 & 8.80 & $\begin{array}{l}\text { Plant is } \\
\text { astringent. } \\
\text { Used for }\end{array}$ & {$[10,12]$} \\
\hline
\end{tabular}


Table 2 Ethnobotanical profile of plants from the area. Plants with novel medicinal uses are highlighted in bold (Continued)

\begin{tabular}{|c|c|c|c|c|c|c|c|c|c|c|c|}
\hline Family & BN. Acc. & $\mathrm{LN}$ & $\mathrm{PU}$ & $\operatorname{Rec}$ & App & Uses & $N$ & UV & $\mathrm{CSI}$ & $\begin{array}{l}\text { Previously } \\
\text { reported uses }\end{array}$ & References \\
\hline & & & & & & $\begin{array}{l}\text { gum sores } \\
\text { and colorize } \\
\text { lips as a } \\
\text { traditional } \\
\text { cosmetic } \\
\text { item. }\end{array}$ & & & & $\begin{array}{l}\text { toothache, bark } \\
\text { is used as chew } \\
\text { stick. Fruits is } \\
\text { edible and sold } \\
\text { in market. }\end{array}$ & \\
\hline \multirow[t]{5}{*}{ Lamiaceae } & $\begin{array}{l}\text { Colebrookea } \\
\text { oppositifolia } \\
\text { Lodd. FK-4999 }\end{array}$ & $\begin{array}{l}\text { Muskana } \\
\text { Bhekar }\end{array}$ & Leaves & Wrap & Topical & $\begin{array}{l}\text { Paste is } \\
\text { wrapped on } \\
\text { injury site. }\end{array}$ & 3 & 0.19 & 2.92 & $\begin{array}{l}\text { Antibacterial, } \\
\text { antifungal }\end{array}$ & [28] \\
\hline & $\begin{array}{l}\text { Mentha } \\
\text { longifolia (L.) L. } \\
\text { FK-5031 }\end{array}$ & $\begin{array}{l}\text { Jangli } \\
\text { pondna }\end{array}$ & Aerial parts & Powder & Oral & $\begin{array}{l}\text { Carminative. } \\
\text { Used as an } \\
\text { ingredient of } \\
\text { sausage }\end{array}$ & 5 & 0.190 & 9.80 & $\begin{array}{l}\text { Used against } \\
\text { diarrhea. Used } \\
\text { as tonic to } \\
\text { cough, flu and } \\
\text { asthma. Anti- } \\
\text { cholera and } \\
\text { anti-dyspepsia } \\
\text { effects. }\end{array}$ & [10] \\
\hline & $\begin{array}{l}\text { Mentha arvensis } \\
\text { L. } \\
\text { FK-5032 }\end{array}$ & Podna & Aerial parts & Sauce & Oral & $\begin{array}{l}\text { Carminative. } \\
\text { Used for its } \\
\text { cooling } \\
\text { effects; } \\
\text { considered } \\
\text { good for } \\
\text { digestive } \\
\text { system. }\end{array}$ & 7 & 0.99 & 9.90 & $\begin{array}{l}\text { Used to cure } \\
\text { bronchial } \\
\text { disorders and } \\
\text { abdominal } \\
\text { pain. }\end{array}$ & [10] \\
\hline & $\begin{array}{l}\text { Micromeria } \\
\text { biflora (Buch - } \\
\text { Ham. ex D. Don) } \\
\text { Benth. FK-5035 }\end{array}$ & & Roots & Infusion & Oral & $\begin{array}{l}\text { Used for a } \\
\text { muscular } \\
\text { itching of the } \\
\text { stomach; } \\
\text { locally known } \\
\text { as "naar". }\end{array}$ & 1 & 0.012 & 3.80 & $\begin{array}{l}\text { Used to treat } \\
\text { kidney stone. } \\
\text { Used as an } \\
\text { herbal tea } \\
\text { ingredient. } \\
\text { Used to treat } \\
\text { toothache. }\end{array}$ & {$[10,45,46]$} \\
\hline & $\begin{array}{l}\text { Otostegia } \\
\text { limbata (Benth), } \\
\text { Boiss. FK-5040 }\end{array}$ & $\begin{array}{l}\text { Chitti } \\
\text { sumbal }\end{array}$ & Aerial parts & Paste & Topical & $\begin{array}{l}\text { Used to cure } \\
\text { open wound } \\
\text { infections }\end{array}$ & 4 & 0.341 & 2.48 & Antibacterial & [47] \\
\hline Lilliaceae & $\begin{array}{l}\text { Tulipa stellata } \\
\text { Hook. FK- } 5073\end{array}$ & Goggar & Bulb & & & $\begin{array}{l}\text { Bulb locally } \\
\text { known as } \\
\text { "gogger" is } \\
\text { edible. }\end{array}$ & 3 & 0.038 & 3.50 & $\begin{array}{l}\text { It has toxic } \\
\text { effects on the } \\
\text { CNS of animals } \\
\text { resulting in } \\
\text { high fever, } \\
\text { abdominal } \\
\text { cramps, violent } \\
\text { tremors and } \\
\text { twitching of } \\
\text { the muscles }\end{array}$ & [43] \\
\hline Lythraceae & $\begin{array}{l}\text { Punica } \\
\text { granatum L. FK- } \\
5048\end{array}$ & Drunni & Fruit peel & Powder & Oral & $\begin{array}{l}\text { Fruit is edible, } \\
\text { used to } \\
\text { prevent } \\
\text { infection and } \\
\text { to heal } \\
\text { wounds. }\end{array}$ & 9 & 0.111 & 5.50 & $\begin{array}{l}\text { Powder made } \\
\text { from fruit is } \\
\text { used for } \\
\text { diabetes and } \\
\text { gastric ulcer. } \\
\text { Ground rind of } \\
\text { dried flower is } \\
\text { given for } \\
\text { leucorrhea. }\end{array}$ & {$[48]$} \\
\hline Malvaceae & $\begin{array}{l}\text { Grewia optiva } \\
\text { Drumm. ex } \\
\text { Burret FK-5018 }\end{array}$ & Tahamman & Aerial parts & Fodder & Oral & $\begin{array}{l}\text { Effective for } \\
\text { childbirth in } \\
\text { cattle. Fruit is } \\
\text { edible. Used } \\
\text { to make } \\
\text { ropes. Used } \\
\text { as forage. }\end{array}$ & 5 & 0.133 & 2.20 & $\begin{array}{l}\text { Used for } \\
\text { smooth } \\
\text { delivery and } \\
\text { quick discharge } \\
\text { of afterbirth, } \\
\text { given to young } \\
\text { animals to } \\
\text { induce puberty. }\end{array}$ & [9] \\
\hline
\end{tabular}


Table 2 Ethnobotanical profile of plants from the area. Plants with novel medicinal uses are highlighted in bold (Continued)

\begin{tabular}{|c|c|c|c|c|c|c|c|c|c|c|c|}
\hline Family & BN. Acc. & LN & PU & $\operatorname{Rec}$ & App & Uses & $N$ & UV & CSI & $\begin{array}{l}\text { Previously } \\
\text { reported uses }\end{array}$ & References \\
\hline \multirow[t]{2}{*}{ Moraceae } & $\begin{array}{l}\text { Broussonetia } \\
\text { papayrifera (L.) } \\
\text { L'Hér. ex Vent. } \\
\text { FK-4993 }\end{array}$ & Jangli Toot & & & & $\begin{array}{l}\text { Used as fuel } \\
\text { and leaves as } \\
\text { fodder. }\end{array}$ & 5 & 0.066 & 4.20 & $\begin{array}{l}\text { The plant is } \\
\text { toxic and } \\
\text { causes allergies. } \\
\text { It is also used } \\
\text { for fodder and } \\
\text { fuel purposes. }\end{array}$ & [1 1 1] \\
\hline & $\begin{array}{l}\text { Ficus palmata } \\
\text { Forrsk } \\
\text { FK-5013 }\end{array}$ & Tussa & Leaf & Ash & Nasal & $\begin{array}{l}\text { Fruit is edible. } \\
\text { Leaves are } \\
\text { used as } \\
\text { fodder, wood } \\
\text { as fuel. Ash of } \\
\text { leaves is } \\
\text { sniffed as } \\
\text { expectorant. }\end{array}$ & 7 & 0.190 & 7.0 & $\begin{array}{l}\text { Latex is applied } \\
\text { on viral warts } \\
\text { present on skin. } \\
\text { Fruit is used to } \\
\text { cure respiratory } \\
\text { disorders }\end{array}$ & [43] \\
\hline Onagraceae & $\begin{array}{l}\text { Oenothera rosea } \\
\text { L'Her ex Aiton. } \\
\text { FK-5039 }\end{array}$ & Nashtar & Whole plant & Infusion & Oral & $\begin{array}{l}\text { Used to treat } \\
\text { whooping } \\
\text { cough and } \\
\text { asthma. }\end{array}$ & 3 & 0.014 & 2.00 & $\begin{array}{l}\text { Used to reduce } \\
\text { thrombosis, } \\
\text { menopause } \\
\text { and other } \\
\text { degenerative } \\
\text { diseases. Plant } \\
\text { is used as } \\
\text { fodder. }\end{array}$ & {$[10,49]$} \\
\hline Oxalidaceae & $\begin{array}{l}\text { Oxalis } \\
\text { corniculata L. } \\
\text { FK-5042 }\end{array}$ & & & & & $\begin{array}{l}\text { Used as } \\
\text { fodder }\end{array}$ & 2 & 0.040 & 0.98 & $\begin{array}{l}\text { Reported for } \\
\text { antioxidant and } \\
\text { antitumor } \\
\text { activity. }\end{array}$ & [50] \\
\hline Pinaceae & $\begin{array}{l}\text { Cedrus deodara } \\
\text { (Roxb. ex } \\
\text { D.Don) G.Don } \\
\text { FK-4996 }\end{array}$ & & & & & $\begin{array}{l}\text { Used as } \\
\text { furniture } \\
\text { wood. }\end{array}$ & 7 & 0.148 & 1.39 & $\begin{array}{l}\text { Used as anti- } \\
\text { inflammatory, } \\
\text { analgesic,anti- } \\
\text { hyperglycemia, } \\
\text { antispasmodic, } \\
\text { insecticidal, } \\
\text { anti-apoptotic, } \\
\text { anti-cancer, } \\
\text { immmuno- } \\
\text { modulatory, } \\
\text { molluscidal, } \\
\text { anxiolytic and } \\
\text { anticonvulsant } \\
\text { properties. }\end{array}$ & [21] \\
\hline \multirow[t]{2}{*}{ Plantaginiaceae } & $\begin{array}{l}\text { Plantago } \\
\text { lanceolata L. FK- } \\
5043\end{array}$ & Batti & aerial parts & $\begin{array}{l}\text { Dried } \\
\text { Husk }\end{array}$ & Oral & $\begin{array}{l}\text { Used for the } \\
\text { treatment of } \\
\text { constipation } \\
\text { and } \\
\text { hemorrhoids. }\end{array}$ & 4 & 0.007 & 0.10 & $\begin{array}{l}\text { Used in } \\
\text { rheumatism } \\
\text { and griping } \\
\text { pain of bowels. } \\
\text { Astringent. } \\
\text { Leaves used in } \\
\text { fevers and } \\
\text { dysentery and } \\
\text { to prevent } \\
\text { prolonged } \\
\text { bleeding after } \\
\text { giving birth. } \\
\text { Chopped } \\
\text { leaves are used } \\
\text { to color skin. }\end{array}$ & {$[10,51-53]$} \\
\hline & $\begin{array}{l}\text { Plantago major } \\
\text { L. FK-5044 }\end{array}$ & Salathee & Leaves & Paste & Oral & $\begin{array}{l}\text { Used for } \\
\text { wound } \\
\text { healing and } \\
\text { constipation. } \\
\text { Used as } \\
\text { fodder also }\end{array}$ & 5 & 0.004 & 2.00 & $\begin{array}{l}\text { Used in } \\
\text { rheumatism } \\
\text { and griping } \\
\text { pain of bowels, } \\
\text { astringent, } \\
\text { leaves. Used in } \\
\text { fevers } \\
\text { dysentery and } \\
\text { to prevent }\end{array}$ & [51] \\
\hline
\end{tabular}


Table 2 Ethnobotanical profile of plants from the area. Plants with novel medicinal uses are highlighted in bold (Continued)

\begin{tabular}{|c|c|c|c|c|c|c|c|c|c|c|c|}
\hline Family & BN. Acc. & $\mathrm{LN}$ & PU & $\operatorname{Rec}$ & App & Uses & $N$ & UV & CSI & $\begin{array}{l}\text { Previously } \\
\text { reported uses }\end{array}$ & References \\
\hline & & & & & & & & & & $\begin{array}{l}\text { prolonged after } \\
\text { birth bleeding. }\end{array}$ & \\
\hline \multirow[t]{4}{*}{ Poaceae } & $\begin{array}{l}\text { Arundo donax L. } \\
\text { FK-5036 }\end{array}$ & $\mathrm{Nal}$ & Whole plant & Pipes & & $\begin{array}{l}\text { Used to make } \\
\text { pipes and } \\
\text { thatching } \\
\text { material. }\end{array}$ & 4 & 0.145 & 4.40 & & \\
\hline & $\begin{array}{l}\text { Cynodon } \\
\text { dactylon (L.) } \\
\text { Pers. FK-5002 }\end{array}$ & & Aerial parts & Paste & Topical & $\begin{array}{l}\text { Paste of aerial } \\
\text { parts is } \\
\text { applied on } \\
\text { broken bones } \\
\text { for healing }\end{array}$ & 5 & 0.133 & 1.20 & . & \\
\hline & $\begin{array}{l}\text { Poa annua L. } \\
\text { FK- } 5046\end{array}$ & Malla & Aerial parts & & & $\begin{array}{l}\text { Grazed on by } \\
\text { cattle. }\end{array}$ & 2 & 0.034 & 1.80 & Used as fodder & [10] \\
\hline & $\begin{array}{l}\text { Setaria pumila } \\
\text { (Poir.) Roem \& } \\
\text { Schult FK- } 5064\end{array}$ & & Whole plant & & & $\begin{array}{l}\text { Used as } \\
\text { fodder }\end{array}$ & 2 & 0.071 & 0.21 & $\begin{array}{l}\text { Chewed for } \\
\text { toothache and } \\
\text { infection. } \\
\text { Powder is } \\
\text { applied for } \\
\text { infection. }\end{array}$ & [54] \\
\hline \multirow[t]{2}{*}{ Polygonaceae } & $\begin{array}{l}\text { Rumex } \\
\text { hastatus D. } \\
\text { Don. FK-5056 }\end{array}$ & Chukhree & Aerial parts & Infusion & Oral & $\begin{array}{l}\text { Used for } \\
\text { jaundice and } \\
\text { antidote for } \\
\text { allergies } \\
\text { caused by } \\
\text { weeds. }\end{array}$ & 3 & 0.051 & 2.40 & $\begin{array}{l}\text { Used as } \\
\text { antirheumatic, } \\
\text { tonic and } \\
\text { laxative. } \\
\text { Antioxidant, } \\
\text { antitumor and } \\
\text { antimicrobial } \\
\text { Antioxidant, } \\
\text { and to cure } \\
\text { dental } \\
\text { problems. }\end{array}$ & {$[55-58]$} \\
\hline & $\begin{array}{l}\text { Rumex } \\
\text { nepalensis } \\
\text { Spreng FK-5057 }\end{array}$ & Khoh & Aerial parts & Infusion & Oral & $\begin{array}{l}\text { Used as } \\
\text { antidote for } \\
\text { allergic } \\
\text { reaction of } \\
\text { other weeds. } \\
\text { Cooked as } \\
\text { vegetable }\end{array}$ & 5 & 0.074 & 3.40 & $\begin{array}{l}\text { Leaf extract is } \\
\text { used as } \\
\text { antiseptic, } \\
\text { cooked as } \\
\text { vegetable. }\end{array}$ & [10] \\
\hline \multirow[t]{2}{*}{ Pteridaceae } & $\begin{array}{l}\text { Adiantum } \\
\text { capillus-veneris. } \\
\text { L. FK-4983 }\end{array}$ & Kakwai & Whole plant & Infusion & Oral & $\begin{array}{l}\text { Infusion of } \\
\text { whole plant is } \\
\text { used to treat } \\
\text { flu and } \\
\text { urinary tract } \\
\text { infection (UTI). }\end{array}$ & 6 & 0.029 & 2.40 & $\begin{array}{l}\text { Used to treat } \\
\text { bronchitis, hair } \\
\text { loss, } \\
\text { inflammatory } \\
\text { and skin } \\
\text { diseases. }\end{array}$ & [59] \\
\hline & $\begin{array}{l}\text { Pteris cretica L. } \\
\text { FK-5045 }\end{array}$ & Koochi & Aerial parts & & & $\begin{array}{l}\text { Used to } \\
\text { clean milk } \\
\text { utensils as } \\
\text { an } \\
\text { antifungal } \\
\text { agent. }\end{array}$ & 2 & 0.083 & 2.40 & $\begin{array}{l}\text { The paste } \\
\text { wrapped in a } \\
\text { bandage is } \\
\text { applied on } \\
\text { External } \\
\text { wounds. }\end{array}$ & [10] \\
\hline \multirow[t]{3}{*}{ Ranunculaceae } & $\begin{array}{l}\text { Clematis grata } \\
\text { Wall FK-5001 }\end{array}$ & & Aerial parts & Decoction & oral & $\begin{array}{l}\text { Aqueous } \\
\text { extract of } \\
\text { aerial parts is } \\
\text { used as to } \\
\text { treat worms. }\end{array}$ & 6 & 0.050 & 6.00 & $\begin{array}{l}\text { Used to treat } \\
\text { intestinal } \\
\text { worms in man } \\
\text { and cattle. }\end{array}$ & [32] \\
\hline & $\begin{array}{l}\text { Ranunculus } \\
\text { laetus Wall. Ex } \\
\text { Royle. FK. } 5051\end{array}$ & Mleeth & aerial parts & Paste & Topical & $\begin{array}{l}\text { Used for skin } \\
\text { conditions, } \\
\text { rashes and } \\
\text { burns. }\end{array}$ & 2 & 0.035 & 2.40 & $\begin{array}{l}\text { Paste of fresh } \\
\text { leaves is } \\
\text { germicidal, } \\
\text { applied once a } \\
\text { day. }\end{array}$ & [3] \\
\hline & $\begin{array}{l}\text { Ranunculus } \\
\text { arvensis L. FK- }\end{array}$ & Chochumba & Aerial parts & Vegetable & Oral & $\begin{array}{l}\text { Used to cure } \\
\text { infections Part }\end{array}$ & 2 & 0.093 & 1.50 & $\begin{array}{l}\text { Used to treat } \\
\text { gout, fever and }\end{array}$ & [23] \\
\hline
\end{tabular}


Table 2 Ethnobotanical profile of plants from the area. Plants with novel medicinal uses are highlighted in bold (Continued)

\begin{tabular}{|c|c|c|c|c|c|c|c|c|c|c|c|}
\hline Family & BN. Acc. & LN & PU & $\operatorname{Rec}$ & App & Uses & $N$ & UV & CSI & $\begin{array}{l}\text { Previously } \\
\text { reported uses }\end{array}$ & References \\
\hline & 5052 & & & & & $\begin{array}{l}\text { of a } \\
\text { traditional } \\
\text { vegetable }\end{array}$ & & & & asthma. & \\
\hline Rhamnaceae & $\begin{array}{l}\text { Ziziphus jujube } \\
\text { Mill. FK- } 5085\end{array}$ & Tukbair & Leaves & Powder & Oral & $\begin{array}{l}\text { Considered } \\
\text { sacred, leaves } \\
\text { and fruit are } \\
\text { used as pain } \\
\text { healers. }\end{array}$ & & 0.091 & 5.50 & $\begin{array}{l}\text { Pain healer, } \\
\text { gum problems, } \\
\text { stomach } \\
\text { disorder, heart } \\
\text { burn, diarrhea, } \\
\text { hemorrhoids, } \\
\text { emoilient, skin } \\
\text { disease }\end{array}$ & {$[60]$} \\
\hline \multirow[t]{6}{*}{ Rosaceae } & $\begin{array}{l}\text { Fragaria vesca L. } \\
\text { FK-5014 }\end{array}$ & $\begin{array}{l}\text { Ammal } \\
\text { budha }\end{array}$ & Fruit & Tea & Oral & $\begin{array}{l}\text { Fruit is edible } \\
\text { and used to } \\
\text { treat heart } \\
\text { burn. Diarrhea } \\
\text { in children is } \\
\text { treated with } \\
\text { leaves. Used } \\
\text { to make tea. }\end{array}$ & 8 & 0.125 & 9.70 & & \\
\hline & $\begin{array}{l}\text { Prunus persica } \\
\text { (L.) Batsch } \\
\text { FK- } 5047\end{array}$ & Arwari & Fruit & & & $\begin{array}{l}\text { Fruit is edible, } \\
\text { wood is used } \\
\text { for fuel. }\end{array}$ & 6 & 0.186 & 8.00 & $\begin{array}{l}\text { Antiseptic, used } \\
\text { for external } \\
\text { wounds. Used } \\
\text { for fuel wood } \\
\text { and fodder }\end{array}$ & [12] \\
\hline & $\begin{array}{l}\text { Pyrus pashia } \\
\text { Buch.-Ham ex } \\
\text { D. Don. FK- } \\
5049\end{array}$ & Tangi & Leaves & Decoction & Oral & $\begin{array}{l}\text { Used for } \\
\text { hepatitis B } \\
\text { and C. Wood } \\
\text { is used in } \\
\text { agriculture for } \\
\text { tools and as } \\
\text { lumber wood. }\end{array}$ & 4 & 0.191 & 4.50 & $\begin{array}{l}\text { Used as forage. } \\
\text { Wood is used } \\
\text { for making } \\
\text { sticks, leaf } \\
\text { extract is used } \\
\text { for hair loss. }\end{array}$ & [10] \\
\hline & $\begin{array}{l}\text { Rosa brunonii } \\
\text { Lindl FK- } 5053\end{array}$ & Jangli gulab & Leaves & Paste & Oral & $\begin{array}{l}\text { Flower is used } \\
\text { against } \\
\text { scabies. Used } \\
\text { as fodder. }\end{array}$ & 4 & 0.098 & 5.40 & $\begin{array}{l}\text { Decoction is } \\
\text { taken internally } \\
\text { for constipation }\end{array}$ & {$[20]$} \\
\hline & $\begin{array}{l}\text { Rubus ellipticus } \\
\text { Sm. FK-5054 }\end{array}$ & Akhray & & & & $\begin{array}{l}\text { Used for } \\
\text { fencing. } \\
\text { Leaves are } \\
\text { used as } \\
\text { fodder. Fruit is } \\
\text { edible. }\end{array}$ & 5 & 0.220 & 8.80 & $\begin{array}{l}\text { The fruits is } \\
\text { edible and } \\
\text { laxative. Used } \\
\text { in fencing and } \\
\text { hedges. }\end{array}$ & {$[61]$} \\
\hline & $\begin{array}{l}\text { Rubus fruticosus } \\
\text { L. FK-5055 }\end{array}$ & Pamnar & $\begin{array}{l}\text { Fruit/ } \\
\text { leaves }\end{array}$ & Food & Oral & $\begin{array}{l}\text { Used for } \\
\text { paling. Fruit is } \\
\text { used for } \\
\text { chilling effect. } \\
\text { Leaves used } \\
\text { as fodder. }\end{array}$ & 5 & 0.066 & 3.50 & $\begin{array}{l}\text { Fruits are } \\
\text { edible and } \\
\text { have cooling } \\
\text { effect. Spiny } \\
\text { branches are } \\
\text { used for } \\
\text { fencing. }\end{array}$ & [10] \\
\hline Rubiaceae & $\begin{array}{l}\text { Rubia manjith } \\
\text { Roxb. ex } \\
\text { Fleming FK- } \\
5063\end{array}$ & Tharyat & & & & $\begin{array}{l}\text { Used as } \\
\text { fodder. }\end{array}$ & 2 & 0.191 & 1.00 & & \\
\hline Rutaceae & $\begin{array}{l}\text { Zanthoxylum } \\
\text { armatum DC. } \\
\text { FK- } 5084\end{array}$ & Timber & $\begin{array}{l}\text { Seed/ } \\
\text { shoots }\end{array}$ & Sauce & Oral & $\begin{array}{l}\text { Shoot is used } \\
\text { to make } \\
\text { tooth stick for } \\
\text { treatment of } \\
\text { oral } \\
\text { infections. }\end{array}$ & 5 & 0.267 & 8.85 & $\begin{array}{l}\text { Ground leaves } \\
\text { used for } \\
\text { digestion. Fruit } \\
\text { is carminative } \\
\text { and is used in } \\
\text { sauce. Fruit is } \\
\text { also used for } \\
\text { piles. }\end{array}$ & [10] \\
\hline Sapindaceae & $\begin{array}{l}\text { Dodonaea } \\
\text { viscoa Jacq. FK- }\end{array}$ & Sanatha & Woody parts & & & $\begin{array}{l}\text { Used as fuel } \\
\text { wood and }\end{array}$ & 7 & 0.430 & 3.80 & & \\
\hline
\end{tabular}


Table 2 Ethnobotanical profile of plants from the area. Plants with novel medicinal uses are highlighted in bold (Continued)

\begin{tabular}{|c|c|c|c|c|c|c|c|c|c|c|c|}
\hline Family & BN. Acc. & LN & PU & $\operatorname{Rec}$ & App & Uses & $N$ & UV & CSI & $\begin{array}{l}\text { Previously } \\
\text { reported uses }\end{array}$ & References \\
\hline & 5005 & & & & & $\begin{array}{l}\text { roof thatching } \\
\text { in mud } \\
\text { houses. }\end{array}$ & & & & & \\
\hline Saxifragraceae & $\begin{array}{l}\text { Bergenia } \\
\text { stracheyi Auct. } \\
\text { FK- } 4991\end{array}$ & Bhatphary & Tuber & Powder & Oral & $\begin{array}{l}\text { The plant is } \\
\text { used to } \\
\text { remove } \\
\text { kidney stone. } \\
\text { Tuber powder } \\
\text { is used for } \\
\text { wound } \\
\text { healing and } \\
\text { diabetes. }\end{array}$ & 3 & 0.014 & 4.00 & $\begin{array}{l}\text { Root extract is } \\
\text { used to cure } \\
\text { ulcers, coughs } \\
\text { and colds. Bark } \\
\text { is antiseptic } \\
\text { and used to } \\
\text { heal cuts and } \\
\text { wounds. }\end{array}$ & [43] \\
\hline Simaroubaceae & $\begin{array}{l}\text { Ailanthus } \\
\text { altissimus (Mill) } \\
\text { Swingle. FK- } \\
4985\end{array}$ & Drave & Woody parts & & & $\begin{array}{l}\text { Used for fuel } \\
\text { and timber. }\end{array}$ & 6 & 0.029 & 3.00 & $\begin{array}{l}\text { Used for timber } \\
\text { and fuel. }\end{array}$ & [27] \\
\hline \multirow[t]{3}{*}{ Solanaceae } & $\begin{array}{l}\text { Solanum } \\
\text { villosum Mill FK- } \\
5060\end{array}$ & Kachmach & $\begin{array}{l}\text { Fruit/ } \\
\text { aerial parts }\end{array}$ & Vegetable & Oral & $\begin{array}{l}\text { Fruit is edible } \\
\text { and effective } \\
\text { for mouth } \\
\text { sores. Cooked } \\
\text { as vegetable } \\
\text { and used to } \\
\text { treat } \\
\text { stomachache. }\end{array}$ & 3 & 0.480 & 5.40 & $\begin{array}{l}\text { Fresh leaves are } \\
\text { applied } \\
\text { Externally for } \\
\text { swelling joints. } \\
\text { Decoction is } \\
\text { drunk for } \\
\text { constipation. }\end{array}$ & {$[62]$} \\
\hline & $\begin{array}{l}\text { Solanum } \\
\text { pseudocapsicum } \\
\text { L. FK-5061 }\end{array}$ & Marcholi & Leaves & Paste & Topical & $\begin{array}{l}\text { Used to treat } \\
\text { boils. } \\
\text { Excessive use } \\
\text { may cause } \\
\text { vomiting. }\end{array}$ & 3 & 0.014 & 0.30 & $\begin{array}{l}\text { Fresh leaves are } \\
\text { applied } \\
\text { externally for } \\
\text { swelling joints. } \\
\text { Decoction is } \\
\text { drunk for } \\
\text { constipation. }\end{array}$ & [10] \\
\hline & $\begin{array}{l}\text { Solanum } \\
\text { surattense Burm. } \\
\text { f. FK- } 5062\end{array}$ & Mokri & Whole plant & Vegetable & Oral & $\begin{array}{l}\text { Cooked as } \\
\text { vegetable } \\
\text { that is } \\
\text { effective for } \\
\text { menstrual } \\
\text { problems. }\end{array}$ & 2 & 0.180 & 0.30 & & \\
\hline Ulmaceae & $\begin{array}{l}\text { Ulmus } \\
\text { wallichiana } \\
\text { Planch. FK- } 5077\end{array}$ & Mannu & Leaves & & & $\begin{array}{l}\text { Used as } \\
\text { fodder. Wood } \\
\text { is used to } \\
\text { make } \\
\text { furniture and } \\
\text { fuel. }\end{array}$ & 4 & 0.170 & 4.20 & $\begin{array}{l}\text { Used as fuel, } \\
\text { and for making } \\
\text { furniture, } \\
\text { branches for } \\
\text { fencing and } \\
\text { thatching. }\end{array}$ & {$[63,64]$} \\
\hline Urticaceae & $\begin{array}{l}\text { Debregeasia } \\
\text { longifolia } \\
\text { (Burm.f.) Wedd. } \\
\text { FK-5004 }\end{array}$ & & Fruit & Food & Oral & $\begin{array}{l}\text { Fruit is used } \\
\text { to treat } \\
\text { bloody } \\
\text { diarrhea. Used } \\
\text { for fodder } \\
\text { and fuel. }\end{array}$ & 3 & 0.110 & 4.20 & $\begin{array}{l}\text { The fruits are } \\
\text { grinded and } \\
\text { are used } \\
\text { against bloody } \\
\text { diarrhea. Used } \\
\text { as fodder. }\end{array}$ & [10] \\
\hline \multirow[t]{2}{*}{ Verbenaceae } & $\begin{array}{l}\text { Verbascum } \\
\text { thapsus L. FK- } \\
5080\end{array}$ & $\begin{array}{l}\text { Jangli } \\
\text { tmako }\end{array}$ & Leaves & Wrap & Topical & $\begin{array}{l}\text { Used in } \\
\text { combination } \\
\text { with } \\
\text { mustard oil } \\
\text { and salt to } \\
\text { treat } \\
\text { physical } \\
\text { trauma. }\end{array}$ & 3 & 0.140 & 2.20 & $\begin{array}{l}\text { Leaves are } \\
\text { smoked to } \\
\text { treat asthma } \\
\text { and sore throat. } \\
\text { Leaves are } \\
\text { applied to } \\
\text { inflamed body } \\
\text { parts }\end{array}$ & {$[65]$} \\
\hline & $\begin{array}{l}\text { Verbena } \\
\text { officinalis L. FK- } \\
5079\end{array}$ & Choro & & & & $\begin{array}{l}\text { Used as } \\
\text { fodder. }\end{array}$ & 2 & 0.037 & 2.50 & & \\
\hline Viburnaceae & $\begin{array}{l}\text { Viburnum } \\
\text { grandiflorum }\end{array}$ & Jammar & $\begin{array}{l}\text { Fruit/ } \\
\text { leavs }\end{array}$ & Infusion & Oral & $\begin{array}{l}\text { Infusion is } \\
\text { used to treat }\end{array}$ & 5 & 0.207 & 1.60 & $\begin{array}{l}\text { Extract of } \\
\text { leaves is used }\end{array}$ & {$[65]$} \\
\hline
\end{tabular}


Table 2 Ethnobotanical profile of plants from the area. Plants with novel medicinal uses are highlighted in bold (Continued)

\begin{tabular}{|c|c|c|c|c|c|c|c|c|c|c|}
\hline Family & BN. Acc. & LN & $P U$ & $\operatorname{Rec}$ & App & Uses & $N$ UV & CSI & $\begin{array}{l}\text { Previously } \\
\text { reported uses }\end{array}$ & References \\
\hline & $\begin{array}{l}\text { Wall. Ex DC. FK- } \\
5081\end{array}$ & & & & & $\begin{array}{l}\text { typhoid fever. } \\
\text { Extract of } \\
\text { flower is used } \\
\text { to treat } \\
\text { respiratory } \\
\text { problems. }\end{array}$ & & & $\begin{array}{l}\text { for whopping } \\
\text { cough and also } \\
\text { to relieve } \\
\text { abdominal } \\
\text { pain. Fruits are } \\
\text { used to treat } \\
\text { constipation. }\end{array}$ & \\
\hline Violaceae & $\begin{array}{l}\text { Viola odorata L. } \\
\text { FK- } 5082\end{array}$ & Gul naksha & Flower & Decoction & Oral & $\begin{array}{l}\text { Decoction has } \\
\text { cooling effect. } \\
\text { Juice is used } \\
\text { to treat } \\
\text { earache. }\end{array}$ & 6 & 7.70 & $\begin{array}{l}\text { Leaves are } \\
\text { taken internally } \\
\text { to treat } \\
\text { respiratory } \\
\text { problems. used } \\
\text { in "kahwa" to } \\
\text { treat cough } \\
\text { and headache. }\end{array}$ & {$[61]$} \\
\hline
\end{tabular}

Abbreviations: BN botanical names and accession number (Acc.), Fam family, LN local name, SN scientific name, $P U$ part used, Re. recipe, App. mode of application, UV use value, CSI cultural significance index, $N$ absolute number of informants

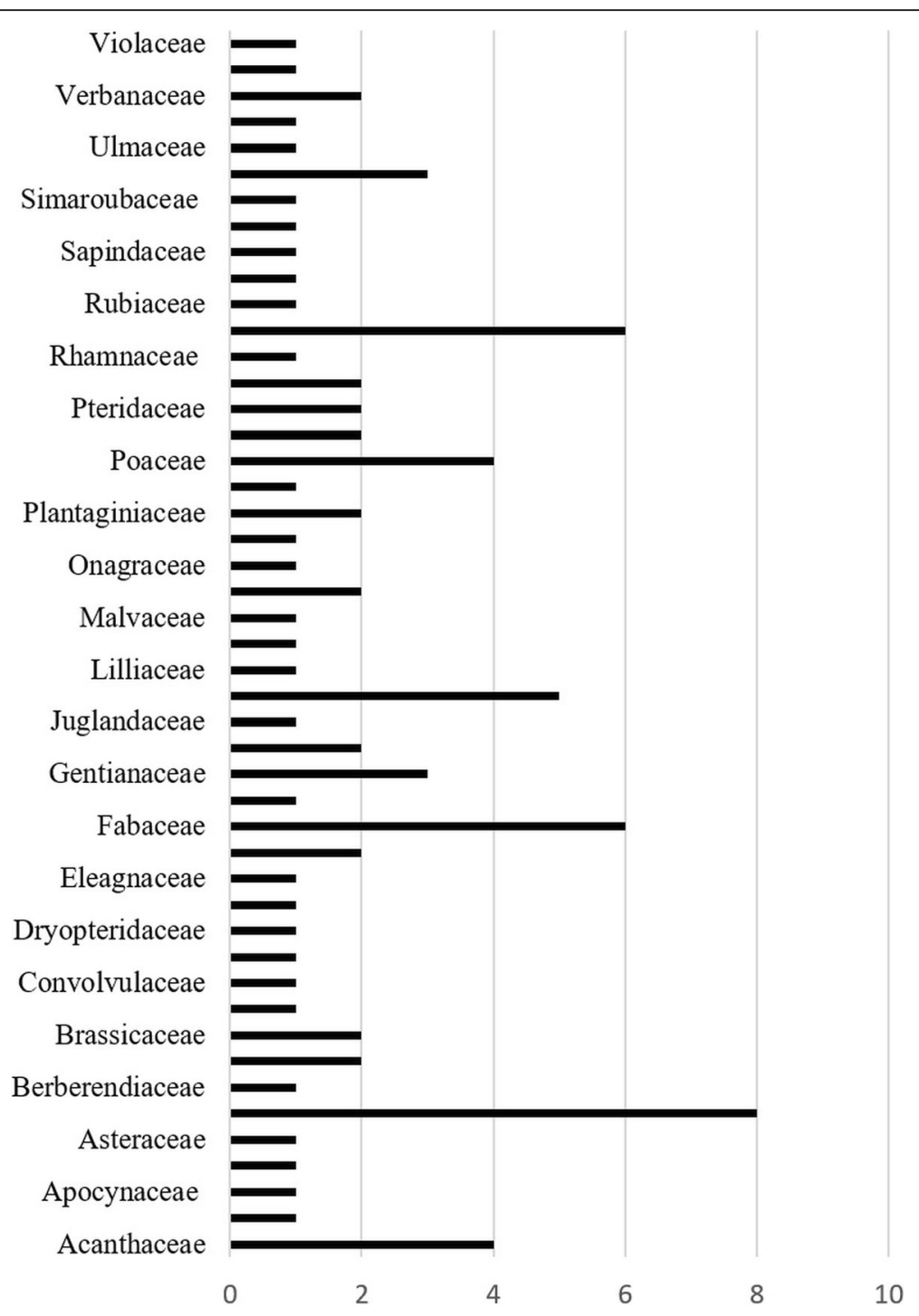

Fig. 2 Family distribution of plants used as ethnomedicine among local communities of District Sudhnoti 


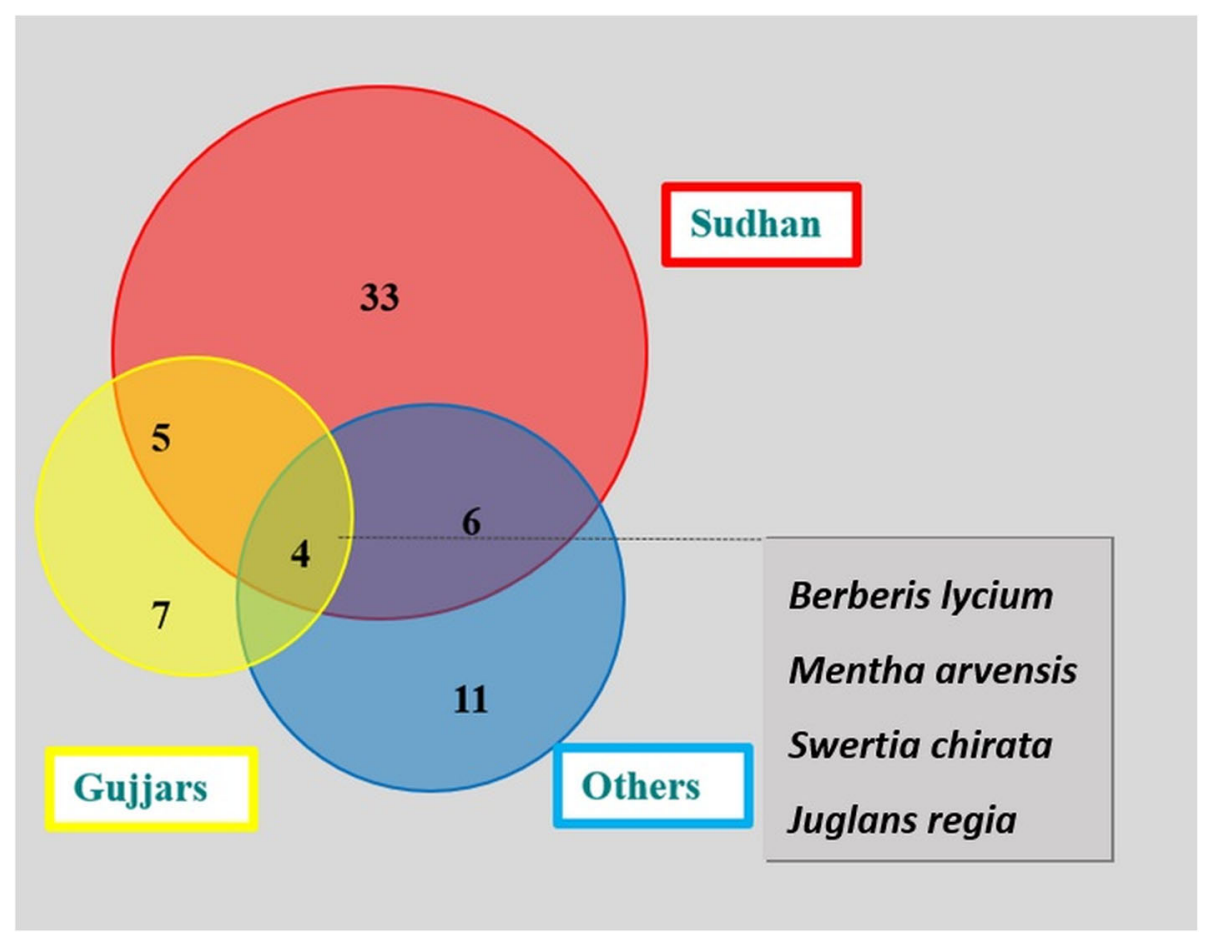

Fig. 3 Venn diagram comparing medicinal plants used by different ethnic groups in Sudhnoti District

\section{Novelty analysis}

The current study's fundamental objective is to compare the findings of the field survey and data analysis with previously reported uses from the region. Comparative analysis identified 11 plant species with specific uses reported from the area for the first time, shown in bold in Table 2. For example, Elaeagnus umbellata was reported for liver ailments, Gentiana olivieri for genital infection, and Pteris cretica for antifungal activity. Pharmacological profiling of plant species was developed with a literature review (Table 2).

\section{Discussion}

The study area has diverse vegetation where 47 plant families represented a field collection of 88 plants. This

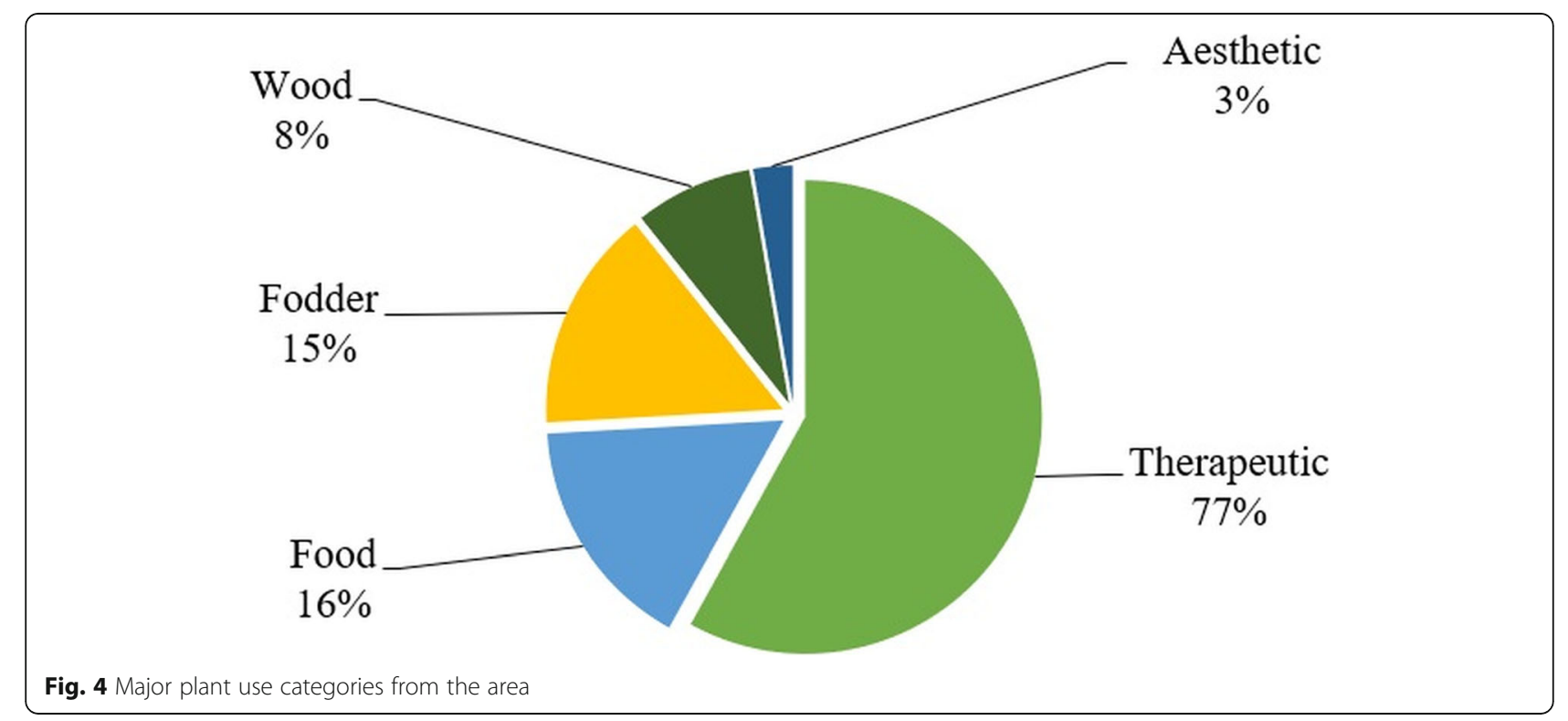



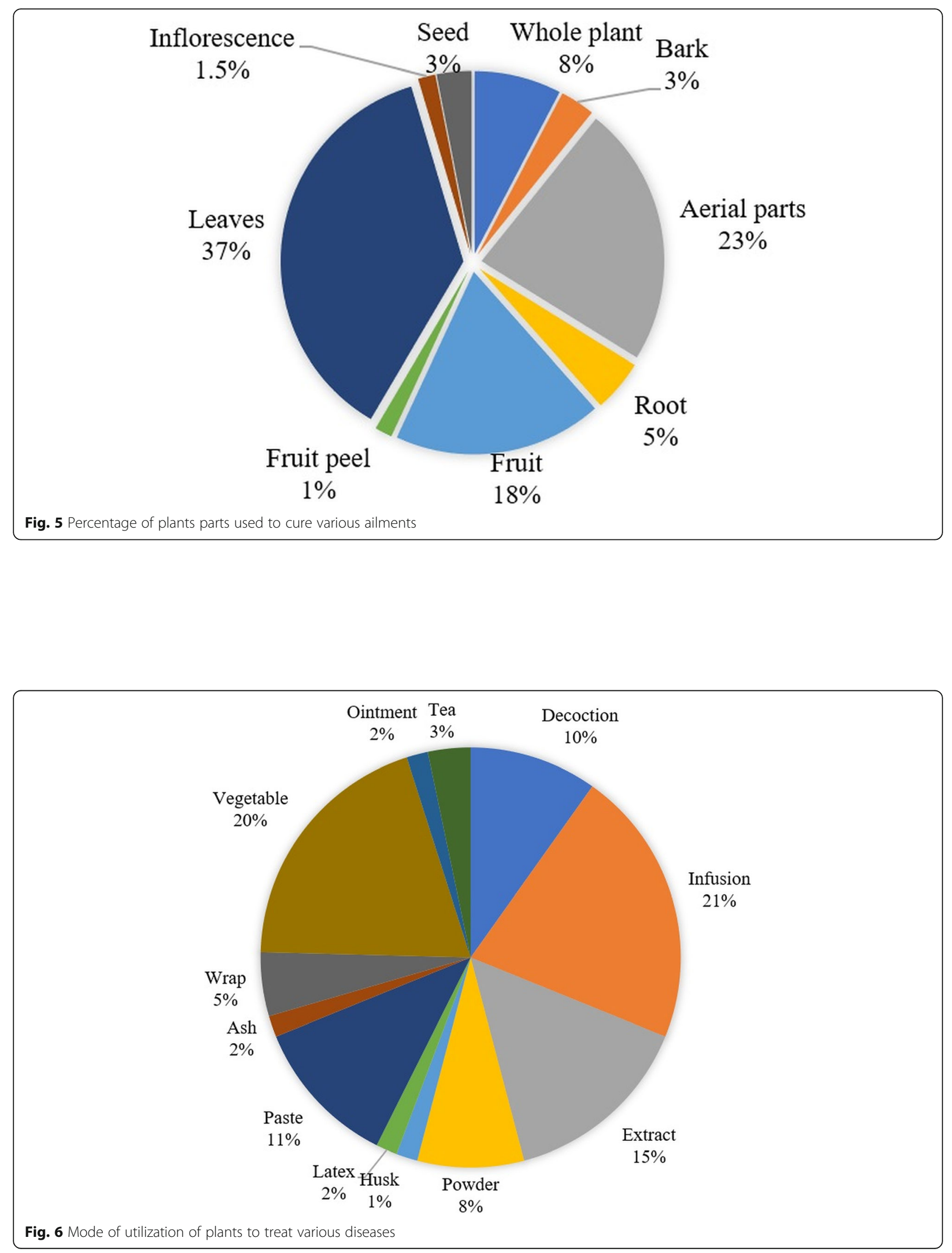
high level of biodiversity was also reported by a previous study conducted in the area [7]. Altitude variation is one of the factors affecting floral richness in the area, as the study area of a mere few thousand $\mathrm{km}^{2}$ represents subtropical (altitude 600 to 2100 m.a.s.l.) to alpine climate conditions. There are rich traditional medicinal practices in the area where $68 \%$ of plant species were used for therapeutic purposes. This indicates the presence of a large number of medicinal plants in the local flora and diverse traditional knowledge. Major ethnic groups such as Sudhan and Gujjar prefer to treat their daily ailments with plant-based remedies. The diversity in traditional knowledge is linked to the fact that both of the major tribes have a strikingly different tribal descent where Sudhans originally migrated from Afghanstan still hold strong ties with Pashtoon folklore. On the other hand, Gujjars, originally migrated from central Asia and northeastern Europe, have a legacy of a nomadic lifestyle and remain settled in alpine Himalayan pastures since hundreds of years.

Moreover, District Sudhnoti is one of the most remote districts of Azad Kashmir, having an inferior transport system and road infrastructure. This disadvantage in communication has a conservatory impact on traditional knowledge as an influx of people with other cultural backgrounds is occurs only occasionally. The majority of the study area population lives in far flung rural areas and manage primary health care needs from traditional means. As easy access to hospitals and allopathic medicines is restricted to a mere few thousand people living around the district headquarters of Sudhnoti and a couple of small towns.

Another reason for the strong belief of the local populace on plant-based remedies is due to the influence of traditional healers, who are often adept in the spiritual component of healing. Generally, healers are wellequipped with the concept of Dam Darood (insufflation) and the mythical practices and prayers accompanying adminstration of herbal formulations. Consequently, they prescribe herbal remedies aided by insufflation accompanied by a period of ardent prayers for that particular ailment. For example, elderly women breathe on the roots of Gentiana oleiveri and advise it to be used for children with genital infections.

Locals use some plant species due to their medicinal or nutraceutical significance mentioned in Holy scriptures such as Quran and Hadith. For example, Ficus is used as an expectorant and Ziziphus as a pain reliever because they are mentioned in Holy texts as sacred plants that can cure a range of human ailments. The combination of traditional and spirtual healing is also reported from other parts of the world as well $[67,68]$.

This study identifies leaves as the most widely used plant part for medicinal purposes. Our findings are in agreement with surveys conducted previously in surrounding areas in the region [32]. The reason for the extensive use of leaves could be their ease of access and having reserves of active metabolites [69, 70]. Here, we found that the preparation of plant-based remedies in infusions and decoctions is the most common form (37\%). Technically, an infusion is different from a decoction in a sense that the former is a liquid preparation in which boiling water is poured on the plant material while the latter is the boiling of plant material in the water [71]. Our findings are supported by previous studies conducted in the Poonch region [32].

Use-value is a basic index that accounts for all possible uses of a plant species. A plant with a high use-value index is often more commonly found and frequently used; examples include Berberis lycium, Zanthoxylum armatum, and Taraxacum officinale. These findings are generally in agreement with previous studies conducted in the are. For example, Berberis lycium has previously been reported as the most valued plant species in the area [8]. Herein, we confirm these findings. B. lycium was also reported as a high use value plant from Kel village of Neelum Valley, Azad Kashmir, Pakistan, where the local communities used a paste and poultice made from this plant for the treatment of jaundice and diarrhea [72]. Higher use-values often contradict the novelty of plant uses, as the most commonly found plant is more frequently cited and valued by a large number of informants than scarcely found novel plants.

Our methodology discouraged the use of reports on plant species involving agronomic or commercial usevalue; this preserved the indices reflection of traditional ethnobotanical knowledge. The cultural significance of a plant considers preference for it, use frequency, and management [18]. Mentha arvensis has the highest CSI value despite having fewer citations due to restricted occurrence along the waterways of high temperate areas. Indeed, local people prefer to use and manage Mentha arvensis for medicinal and food purposes.

In recent years, plant use information has been compared by using statistical tools such as the Jaccard index. Relevance of these tools is, however, a topic of debate for ethnobotanical studies. It was noted that if plant use information documented from a particular area is subjected to a robust review and comparison with previously published reports on that taxon, its novelty is largely established [73].

The present study reports novel medicinal uses of 11 plant species (Table 2, in bold). These plant species were subjected to a robust pharmacological review from other parts of the world (Table 3). In our study, for example, A. millefolium is reported for gum soreness and kidney stones. The pharmacological review of this plant shows its application as an antispasmodic, diuretic, urinary 
antiseptic, and anticancer therapy [74]. Dicliptera roxburghiana is given to cattle for the amelioration of "Takko," an ailment characterized by severe thirst, dehydration, and lethargy. One of the causes of "Takko" as per the general perception of Gujjar (nomadic) tribesmen is the prolonged exposure of cattle to sunlight. Another novel use is the oral intake of Elaegnus umbellata fruit for hepatitis B. Informants in our study considered hepatic disorder as a sign of heating up of the liver and stomach. Therefore, they take fruits of E. umbellata as a cooling agent that potentially has some antiinflammatory or antiviral effects. The plant is otherwise known for pharmacological activities such as antimicrobial [75], phytotoxic [76], antioxidant, and antiproliferative effects [77]. Ethnomedicinally, it has been reported to treat cardiac problems, bacterial infections, and as an astringent in Malaysia and Pakistan [39].

Gentiana olivieri is another plant reported here for a novel use that reduces genital infections and inflammation in male children. The symptoms of this condition include inflammation of the testicles followed by itching and soreness. Fresh flowers are directly squeezed into the mouth in the form of drops as an oral dosage. Additionally, the dried root segments are enveloped in a piece of cloth and advised to be worn around the neck for spiritual healing effects. Previously, it has been reported for hepatoprotective activities in Turkish folk medicine [96]. Pharmacological applications against epilepsy [79], for radical scavenging [80], and as immunomodulatory and hepatoprotective agent [81] have also been reported.

An infusion made from dried leaves of Hedera helix is used to treat diabetes. Previously, it has been reported for treating benign warts [25]. Pharmacologically, it has antibacterial, antifungal, antioxidant [82], anthelmintic [83], anticancer [84], antiviral [85, 86], and antileishmanial [87] activities.

Nerium oleander, a well-known plant for its poisonous effects due to the presence of cardiac glycosides, was reported for diabetes and oral hygiene. In the traditional treatment of diabetes, leaf powder is mixed with the powder of Stewia species to neutralize its toxicity and improve the taste. A young shoot is used as a chewing stick (Miswak) to cure gum infections. Previously, N. oleander has been reported for stomach and earache [22]. Anticancer and antimicrobial activities have been reported for this species [88].

Pteris cretica, a fern, is locally used to clean the milk utensils. It is considered useful for reducing foul odors, but it is also believed that the plant species is a cleansing agent purging milk utensils in a religious context. It is important to note that milk (particularly cow's milk) is considered a holy food item in Hindu culture. Hindus have long inhabited the study area, and therefore, imprints of traditional Indian culture are still evident. Previously, this species has been reported for wound healing applications [10], and it exhibits antimicrobial, antioxidant [89], anti-inflammatory, and anticancer activities [97].

Pyrus pashia is one of many wild fruit yielding species of Rosaceae family. Young apices (with leaflets) of the plant are ground, and the aqueous extract is taken orally for hepatitis $B$ and $C$, colloquially known as "black jaundice." In previous studies, the plant was reported for the treatment of baldness [10]. In vitro bioactivities, such as antimicrobial and antioxidant, have also been reported [89].

Sarcocca saligna locally known as "naidroon" is another species reported here for applications in treating toothache and fevers. Roots of the plant are crushed in water, and the filtered extract is used in the form of drops onto the affected teeth. In fever, particularly one that of enteric origin (Typhoid), the extract is diluted and taken orally. Previously the plant has been reported for treating hypertension [36]. Pharmacological reports include antimicrobial, antispasmodic [90], cardio suppressant, vasodilatory [91], and hyperglycemic effects [98].

Table 3 Pharmacological activities (review) of the plant species with novel therapeutic uses

\begin{tabular}{ll}
\hline Plant name & Pharmacological activities \\
\hline Achillea millefolium & Antispasmaodic, diuretic, urinary antiseptic, antimutagenic, and in the treatment of hyperpigmentation of the skin [74]. \\
Dicliptera roxburghiana & Antioxidant [78]. \\
Elaeagnus umbellata & Aantimicrobial [75], phytotoxic [76], and antioxidant and antiprofilative [77]. \\
Gentiana olivieri & Against epilepsy [79], radical scavanging [80], and immunomodulatory and hepatoprotective [81]. \\
Hedera helix & Antibacterial, antifungal and antioxidant [82], anthelmintic [83], anticancer [84], antiviral [85, 86], and antileishmanicidic [87]. \\
Nerium oleander & Antibacterial and anticancer [88]. \\
Pteris cretica & Antimicrobial and antioxidant activities [89]. \\
Pyrus pashia & Antimicrobial and antioxidant \\
Sarcococca saligna & Antimicrobial, antispasmodic [90], hypertension [36], and cardiosuressant and vassodilator [91]. \\
Rumex hastatus & Antipyretic and antiinflammatory [53]. \\
Verbascum thapsus & Antiviral [92-94] and anticancer [95]. \\
\hline
\end{tabular}


A leaf infusion of Rumax hastatus is reported here for liver ailments, particularly jaundice, owing to its cooling effect. Local healers consider jaundice a sign of an "internal heating" and a forthcoming indication of chronic hepatic disorders. Therefore, $R$. hastatus is taken as a chilling agent that "cools down" the body and helps the liver to alleviate the damage. The plant is also reported as an antidote for allergic reactions to weeds such as Parthenium hysterophorus. Here, fresh leaves of $R$. hastatus are reported to be rubbed on the affected body parts. $R$. hastatus has been previously reported for antirheumatic activity, as a tonic, laxative [55], and as an antioxidant, antitumor, and antimicrobial agent [56, 57].

Leaves of Verbascum thapsus are reported here for healing physical trauma; briefly, the fresh leaf is coated with salt sprinkles and wrapped around the injury site to as an analgesic and to heal contusions. $V$. thapsus is commonly known as a wild form of tobacco and smoked at times with a recreational sense. It has also been reported in the region for asthma, sore throat, and inflammation [65]. It is also reported to have anti-lice and flea activity and used for earache [99]. Pharmacological evaluation has consistently established antiviral activities of $V$. thapsus [92-94]. The plant is also reported for anticancer activity [95].

On the other hand, uses reported from other parts of the world are similar to what we report here, such as the antimalarial use of Melia azedarach [45, 46], indicating a global consensus. This phenomenon allows for different cultures to learn from each other about further medicinal uses of their plants and reveals to researchers more bioactivities that some phytochemicals may exerte. Lastly, we conclude that the new traditional ethnopharmacological applications reported here for 11 plant species should be pursued within the framework of ethnobotanical drug discovery. Significant antibacterial activity of plant species including $Z$. armatum, $A$. capillus venaris, $M$. annua, and $A$. absintium has already been reported by our research group [100].

\section{Conclusions}

This is the first detailed ethnobotanical study conducted in District Sudhnoti of Azad Kashmir. A total of 88 plants belonging to 47 families were reported, verifying the high biodiversity of medicinal species in Sudhnoti and, therefore, the need to preserve it. Moreover, 11 plant species were found to have novel uses not reported elsewhere. Information concerning the ethnomedicinal uses of plants, specific plant parts, and application methods is critical to both the preservation of traditional knowledge and provides a basis for future drug discovery activities. In this context, the current study enriches our knowledge basis of the medicinal plant potential and traditional knowledge of District Sudhnoti. Future ethnobotanical and phytochemical research will be fundamental to edxploring the pharmacological potential of these and other botanical traditions.

\section{Supplementary Information}

The online version contains supplementary material available at https://doi. org/10.1186/s13002-021-00435-2.

Additional file 1. Digital images of the collected herbarium specimens.

\section{Acknowledgements}

We thank all of the study participants who generously shared their time and knowledge of local plants with us. We also thank Dr. Sajjad Hussain (Department of Botany, University of Poonch) for assistance with plant identification.

\section{Authors' contributions}

F. K: study design, field surveys, and manuscript preparation. Z. M: study design and supervision. A. M: data analysis, editing, and revision. R. Q: plant identification and revision. R. S: manuscript revision. K. S. A: manuscript revision. C. Q: data analysis, editing, and revision. The authors read and approved the final manuscript

\section{Funding}

Not applicable.

Availability of data and materials

All data generated or analyzed during this study are included in this published article and its supplementary information files.

Ethics approval and consent to participate

All participants gave full oral consent for the study, including presentation of data in a formal publication. Plant specimens were collected for herbarium voucher deposit at of PMAS Arid Agriculture University following standard collection guidelines.

Consent for publication

Not applicable.

\section{Competing interests}

The authors declare that they have no competing interests.

\section{Author details}

${ }^{1}$ Department of Botany, Faculty of Basic and Applied Sciences, University of Poonch Rawalakot, Rawalakot 12350, Pakistan. ${ }^{2}$ Department of Botany, PMAS Arid Agriculture University, Rawalpindi, Pakistan. ${ }^{3}$ Center for the Study of Human Health, Emory University, 550 Asbury Circle, Atlanta, GA 30322, USA.

Received: 13 December 2020 Accepted: 26 January 2021

Published online: 20 March 2021

References

1. Sheng-Ji P. Ethnobotanical approaches of traditional medicine studies: some experiences from Asia. Pharm Biol. 2001;39(sup1):74-9.

2. Akhtar N, Rashid A, Murad W, Bergmeier E. Diversity and use of ethnomedicinal plants in the region of Swat. North Pakistan. J Ethnobiol Ethnomed. 2013;9:25.

3. Shah GM, Abbasi AM, Khan N, Guo X, Khan AM, Hussain M, Bibi S, Nazir A, Shaheen H, Nazir J, Firdous SS, Khalid AU. Cosmetic ethnobotany practiced by tribal women of Kashmir Himalayas. Avicenna J Phytomed. 2014;4(4): 239-50.

4. Ahmad KS, Qureshi R, Hameed M, Ahmad F, Nawaz T. Conservation assessment and medicinal importance of some plants resources from Sharda, Neelum Valley, Azad Jammu and Kashmir, Pakistan. Int J Agr Biol. 2012:14(6):997-1000

5. Martkoplishvili I, Kvavadze E. Some popular medicinal plants and diseases of the Upper Palaeolithic in Western Georgia. Ethnopharmacol. 2015;166:42-52. 
6. Gallahar BHT. Important Indices in Ethnobotany. Ethnobot. Res App. 2007;5: 201-18.

7. Weckerle CS, de Boer HJ, Puri RK, van Andel T, Bussmann RW, Leonti M. Recommended standards for conducting and reporting ethnopharmacological field studies. J Ethnopharmal. 2018;210:125-32.

8. Ishtiaq M, Mahmood A, Maqbool M. Indigenous knowledge of medicinal plants from Sudhnoti district (AJK), Pakistan. J Ethnopharmacol. 2015;168:201-7.

9. Ahmed E, Arshad M, Saboor A, Qureshi R, Mustafa G, Sadiq S, Chaudhari SK. Ethnobotanical appraisal and medicinal use of plants in Patriata, New Murree, evidence from Pakistan. J Ethnobiol Ethnomed. 2013;9:13.

10. Amjad MS, Arshad M, Qureshi R. Ethnobotanical inventory and folk uses of indigenous plants from Pir Nasoora National Park, Azad Jammu and Kashmir. Asian Pac J Trop Biomed. 2015;5(3):234-41.

11. Ajaib M, Zaheer-UD-Din K. Ethnobotanical studies of useful trees of District Kotli, Azad Jammu and Kashmir. Biologia Pak. 2014;60:63-71.

12. Khan MA, Khan MA, Hussain M, Ghulam GM. An ethnobotanical inventory of Himalayan region Poonch valley Azad Kashmir (Pakistan). Ethnobot Res Appl. 2010;8:107-23.

13. International Society of Ethnobiology. 2006. International Society of Ethnobiology Code of Ethics (with 2008 additions). http//ethnobiology.net/code-of-ethics/

14. Nasir E. Flora of Pakistan, Agriculture Research Conference, 1981, Islamabad (Pakistan), 24-27 Oct. 1981. PARC.

15. The Plant List. Version 1.1. Published on the Internet; http://www. theplantlist.org/. (Accessed 1st January). 2013.

16. Turner NJ. "The Importance of a Rose": evaluating the cultural significance of plants in Thompson and Lillooet interior Salish. Am Anthropol. 1988;90:272-90.

17. Da Silva VA, Andrade LDHC, De Albuquerque UP. Revising the cultural significance index: the case of the Fulni-ô in northeastern Brazil. Field Methods. 2006;18(1):98-108.

18. Hoffman B, Gallaher T. Importance indices in ethnobotany. Ethnobot Res Appl. 2007;5:201-18.

19. Sop TK, Oldeland J, Bognounou F, Schmiedel U, Thiombiano A. Ethnobotanical knowledge and valuation of woody plants species: a comparative analysis of three ethnic groups from the sub-Sahel of Burkina Faso. Environ Develop Sustain. 2012;14(5):627-49.

20. Abbasi AM, Khan SM, Ahmad M, Khan MA, Quave CL, Pieroni A. Botanical ethnoveterinary therapies in three districts of the Lesser Himalayas of Pakistan. J Ethnobiol Ethnomed. 2013;9:84. https://doi.org/10.1186/1746-4269-9-84.

21. Chaudhary AK, Ahmad S, Mazumder A. Cedrus deodara (Roxb.) Loud:: A review on its ethnobotany, phytochemical and pharmacological profile. Pharmacog J. 2011;3(23):12-7.

22. Mahmood A, Mahmood A, Shaheen H, Qureshi RA, Sangi Y, Gilani SA. Ethno medicinal survey of plants from district Bhimber Azad Jammu and Kashmir, Pakistan. J Med Plants Res. 2011:5(11):2348-60.

23. Afzal S, Afzal N, Awan MR, Khan TS, Khanum AG, Tariq S. Ethno-botanical studies from Northern Pakistan. J Ayub Med Coll Abbottabad. 2009;21 (1):52-7.

24. Ashraf M, Hayat MQ, Jabeen S, Shaheen N, Khan MA, Yasmin G. Artemisia L. species recognized by the local community of the northern areas of Pakistan as folk therapeutic plants. J Med Plants Res. 2010:4(2):112-9.

25. Ajaib M, Khan Z-u-D, Khan N, Wahab M. Ethnobotanical studies on useful shrubs of district Kotli, Azad Jammu \& Kashmir, Pakistan. Pak J Bot. 2010; 42(3): 1407-15.

26. Rai A. The antiinflammatory and antiarthritic properties of ethanol extract of Hedera helix. Ind J Pharm Sci. 2013;75(1):99-102. https://doi.org/10.4103/ 0250-474X.113537.

27. Barkatullah IM, Hussain F. Ethnobotanical studies of plants of Charkotli Hills, Batkhela District, Malakand, Pakistan. Front Biol China. 2009;4(4):539. https:// doi.org/10.1007/s11515-009-0045-2.

28. Hussain I, Bano A, Ullah F. Traditional drug therapies from various medicinal plants of central karakoram national park, Gilgit-Baltistan Pakistan. Pak J Bot. 2011:43:79-84.

29. Ahmed T, Kanwal R, Hassan M, Ayub N. Assessment of antibacterial activity of Colebrookia oppositifolia against waterborne pathogens isolated from drinking water of the Pothwar region in Pakistan. Hum Ecol Risk Assess. 2009;15(2):401-15.

30. Abbasi AM, Khan MA, Ahmad M, Zafar M, Jahan S, Sultana S. Ethnopharmacological application of medicinal plants to cure skin diseases and in folk cosmetics among the tribal communities of North-West Frontier Province, Pakistan. J Ethnopharmacol. 2010;128(2):322-35. https:/doi.org/10.1016/j.jep.2010.01.052.

31. Bhatia $\mathrm{H}$, Manhas $\mathrm{R}$, Kumar K, Magotra R. Some new additions to the poisonous plant flora of the World. J Biosphere. 2013;2(1):74-7.
32. Amjad MS, Qaeem MF, Ahmad I, Khan SU, Chaudhari SK, Zahid MN, Shaheen $\mathrm{H}$, Khan AM. Descriptive study of plant resources in the context of the ethnomedicinal relevance of indigenous flora: a case study from Toli Peer National Park, Azad Jammu and Kashmir, Pakistan. PLoS One. 2017. 12(2):e0171896

33. Joshi K. Cynoglossum L. A review on phytochemistry and chemotherapeutic potential. J Pharmacgn Phytochem. 2016;5(4):32.

34. Awan AA, Akhtar T, Dar MEUI. Fodder plants of some selected areas of Jhelum Valley. Pak J Biol Sci. 2007;10(9):1547-9.

35. Joshi AR, Joshi K. Indigenous knowledge and uses of medicinal plants by local communities of the Kali Gandaki Watershed Area, Nepal. J Ethnopharmacol. 2000;73(1):175-83.

36. Ahmad L, Semotiuk A, Zafar M, Ahmad M, Sultana S, Liu QR, Zada MP, UI Abidin SZ, Yaseen G. Ethnopharmacological documentation of medicinal plants used for hypertension among the local communities of DIR Lower, Pakistan. J Ethnopharmacol. 2015;175:138-46.

37. Ajaib M, Anjum M, Malik NZ, Sidiqui MF. Ethnobotanical study of some plants of Darguti, tehsil Khuiratta, Azad Jammu and Kashmir. Int J Biol Res. 2015;3(2):101-7.

38. Ahmed KS, Habib S. Indigenous knowledge of some medicinal plants of Himalayan Region, Dowarian Region Neelum Vally, Azad Kashmir, Pakistan Univer J Plant Sci. 2014;2(2):40-7 doi:10.13189.

39. Sarver J, Kumar S, Khan M, Ara M, Anand V. Diversity, distribution and utilization pattern of economically important woody plants associated with agro-forestry in district Rajouri, J \& K (Northwest Himalaya). Ethnobotanical Leaflets. 2009;6:13.

40. Jabbar A, Raza MA, labal Z, Khan MN. An inventory of the ethnobotanicals used as anthelmintics in the southern Punjab (Pakistan). J Ethnopharmacol. 2006;108(1):152-4. https://doi.org/10.1016/j. jep.2006.04.015.

41. Khan MA, Khan MA, Hussain M, Mujtaba G. Medicinal plants used in folk recipes by the inhabitants of himalayan region Poonch Valley Azad Kashmir (Pakistan). J Basic Appl Sci. 2012;8:35-45.

42. Ali H, Qaiser M. The ethnobotany of Chitral valley, Pakistan with particular reference to medicinal plants. Pak J Bot. 2009;41:2009-41.

43. Shaheen H, Nazir J, Firdous SS, Khalid AU. Cosmetic ethnobotany practiced by tribal women of Kashmir Himalayas. Avi J Phytomed. 2014; 4(4):239-50.

44. Kumar R, Bhagat N. Ethnomedicinal plants of district Kathua (J\&K). J Ethnopharmacol. 2012;171:12-27.

45. Mukungu N, Abuga K, Okalebo F, Ingwela R, Mwangi J. Medicinal plants used for management of malaria among the Luhya community of Kakamega East sub-County, Kenya. J Ethnopharmacol. 2016;194:98-107.

46. Nathan SS, Savitha G, George DK, Narmadha A, Suganya L, Chung PG. Efficacy of Melia azedarach L. extract on the malarial vector Anopheles stephensi Liston (Diptera: Culicidae). Bioresour Technol. 2006;97(11): 1316-23.

47. Anwar N, Salik S, Ahmad D. Antibacterial activity of Otostegia limbata. Int Agric Biol. 2009;11:647-50.

48. Khan MA, Khan MH. Ethnoveternary medicinal uses of plants of Poonch Vally. Pak J Weed Sci Res. 2012;18(4):496-507.

49. Hussain S, Hameed A, Ahmad KS, Mahmood A, Nawaz F, Ahmad H. Quantitative ethnopharmacological profiling of medicinal shrubs used by indigenous communities of Rawalakot, District Poonch, Azad Jammu and Kashmir, Pakistan. Rev Bras Farmacogn. 2019;29(5):665-76.

50. Kathiriya A, Das K, Kumar E, Mathai K. Evaluation of antitumor and antioxidant activity of Oxalis corniculata L. Against ehrlich ascites carcinoma on mice. Iran J Cancer Prev. 2010;3(4):157-65.

51. Qureshi R, Waheed A, Arshad M, Umbreen T. Medico-ethnobotanical inventory of tehsil Chakwal, Pakistan. Pak J Bot. 2009;41(2):529-38.

52. Mahmood A, Mahmood A, Malik RN. Indigenous knowledge of medicinal plants from Leepa valley, Azad Jammu and Kashmir, Pakistan. J Ethnopharmacol. 2012;143(1):338-46. https://doi.org/10.1016/j.jep.2012.06.046.

53. Singh S, Kaur R, Sharma SK. Antinociceptive, antiinflammatory and antipyretic activities of Rumex hastatus D. don stem and roots. Pharm Sin. 2013:4(3):95-102.

54. Saghir IA, Awan AA, Majid S. Ethnobotanical studies of Chikar and its allied areas of District Muzaffarabad. Online J Biol Sci. 2001;1(12):1165-70.

55. Shinwari ZK, Gilani SS. Sustainable harvest of medicinal plants at Bulashbar Nullah, Astore (Northern Pakistan). J Ethnopharmacol. 2003; 84(2-3):289-98. 
56. Ahmad S, Ullah F, Ayaz M, Sadiq A, Imran M. Antioxidant and anticholinesterase investigations of Rumex hastatus D. Don: potential effectiveness in oxidative stress and neurological disorders. Biol Res. 2015;48:20

57. Sahreen S, Khan MR, Khan RA, Hadda TB. Evaluation of phytochemical content, antimicrobial, cytotoxic and antitumor activities of extract from Rumex hastatus D. Don roots. BMC Complement Altern Med. 2015;15:211.

58. Rahman IU, ljaz F, lqbal Z, Afzal A, Ali N, Afzal M, Khan MA, Muhammad S, Qadir G, Asif M. A novel survey of the ethno medicinal knowledge of dental problems in Manoor Valley (Northern Himalaya), Pakistan. J Ethnopharmacol. 2016:194:877-94.

59. Vadi R, Manisha V, Swati K. Hansraj (Adiantum capillus veneris linn.): a systematic review on its ethnobotany, phytochemical and pharmacological profile. Int J Ayurveda Pharma Res. 2017;5(6):5-21.

60. Dafni A, Levy S, Lev E. The ethnobotany of Christ's Thorn Jujube (Ziziphus spina-christi) in Israel. J Ethnobiol Ethnomed. 2005:1(1):8.

61. Khan MA, Khan SA, Qureshi MA, Ahmed G, Khan MA, Hussain M, Ghulam GM. Ethnobotany of some useful plants of Poonch Valley Azad Kashmir. J Med Plant Res. 2011;5(26):6140-51.

62. Abbasi AM, Khan MA, Shah MH, Shah MM, Pervez A, Ahmad M. Ethnobotanical appraisal and cultural values of medicinally important wild edible vegetables of Lesser Himalayas-Pakistan. J Ethnobiol Ethnomed. 2013; $9(1): 66$.

63. Ibrar M, Hussain F, Sultan A. Ethnobotanical studies on plant resources of Ranyal hills, District Shangla, Pakistan. Pak J Bot. 2007;39(2):329.

64. Qureshi RA, Ghufran MA, Gilani SA, Sultana K, Ashraf M. Ethnobotanical studies of selected medicinal plants of Sudhan Gali and Ganga Chotti hills, district Bagh, Azad Kashmir. Pak J Bot. 2007;39(7):2275-83.

65. Khan Z, Khuroo A, Dar G. Ethnomedicinal survey of Uri, Kashmir Himalaya. Ind J Tradit Know. 2004;3(4):351-7.

66. Asif M. A brief study of toxic effects of some medicinal herbs on kidney. Adv Biomed Res. 2012;1:44.

67. van der Watt ASJ, van de Water T, Nortje G, Oladeji BD, Seedat S, Gureje O. The perceived effectiveness of traditional and faith healing in the treatment of mental illness: a systematic review of qualitative studies. Soc Psychiatr Psychiatr Epidemiol. 2018;53(6):555-66. https://doi.org/10.1007/s00127-018-1519-9.

68. Kamsani K, Franco FM, Slik F. Comparative account of the traditional healing practices of healers and non-healers in the Kiudang area of Brunei Darussalam. J Ethnopharmacol. 256:112788. https://doi.org/10.1016/j.jep. 2020.112788.

69. Ahmad S, Ullah F, Ayaz M, Zeb A, Ullah F, Sadiq A. Antitumor and antiangiogenic potentials of isolated crude saponins and various fractions of Rumex hastatus D. Don. Biol Res. 2016;49:18.

70. Bieski IGC, Leonti M, Arnason JT, Ferrier J, Rapinski M, Violante IMP, Balogun SO, Pereira JFCA, Figueiredo RDCF, Lopes CRAS, Da Silva DR. Ethnobotanical study of medicinal plants by population of valley of Juruena region, legal Amazon, Mato Grosso, Brazil. J Ethnopharmacol. 2015;173:383-423.

71. World Health Organization \& WHO Expert Committee on Specifications for Pharmaceutical Preparations. Fifty-second report of the WHO Expert Committee on Specifications for Pharmaceutical Preparations. World Health Organization; 2018. https://apps.who.int/iris/handle/10665/272452. License: CC BY-NC-SA 3.0 IGO

72. Ahmad KS, Hamid A, Nawaz F, Hameed M, Ahmad F, Deng J, Akhtar N, Wazarat A, Mahroof S. Ethnopharmacological studies of indigenous plants in Kel village, Neelum Valley, Azad Kashmir, Pakistan. J Ethnobiol Ethnomed. 2017;13:68.

73. Jamshidi-Kia F, Lorigooini Z, Amini-Khoei H. Medicinal plants: past history and future perspective. J Herb Med Pharmacol. 2018;7(1):1-7.

74. Bais S. Review on phytochemical and pharmacological activity of yarrow (Achilla millefolium L.). Der Pharma Chemica. 2017:9(23):89-96.

75. Yoshizawa Y, Kawaii S, Sato T, Murofushi N, Nishimura H. Anticancer and antihypertensive effects of small fruit juices. ACS Symp Ser. 2001;871:8-23.

76. Sabir MS, Ahmad DS, Hussain IM, Tahir KM. Antibacterial activity of Elaeagnus umbellata (Thunb.) a medicinal plant from Pakistan. Saudi Med J. 2007;28(2):259-63.

77. Wang SY, Bowman L, Ding M. Variations in free radical scavenging capacity and antiproliferative activity among different genotypes of autumn olive (Elaeagnus umbellata). Planta Med. 2007;73(5):468-77.

78. Ahmad B, Khan M, Shah NA, Khan RA. In vitro antioxidant potential of Dicliptera roxburghiana. BMC Complement Altern Med. 2013;13(1):140.

79. Aslan M, Orhan DD, Orhan N. Effect of Gentiana olivieri on experimental epilepsy models. Pharmacogn Mag. 2011;7(28):344-9.
80. Orhan N, Hocbac S, Orhan DD, Asian M, Ergun F. Enzyme inhibitory and radical scavenging effects of some antidiabetic plants of Turkey. Iran J Basic Med Sci. 2014;17(6):426-32.

81. Singh S, Yadav CP, Noolvi MN. Immunomodulatory activity of butanol fraction of Gentiana olivieri Griseb. on Balb/C mice. Asian Pac J Trop Biomed. 2012;2(6):433-7.

82. Lans C, Turner N, Khan T, Brauer G, Boepple W. Ethnoveterinary medicines used for ruminants in British Columbia, Canada. J Ethnobiol Ethnomed. 2007;3:11.

83. Eguale T, Tilahun G, Debella A, Feleke A, Makonnen E. Haemonchus contortus: in vitro and in vivo anthelmintic activity of aqueous and hydroalcoholic extracts of Hedera helix. Exp Parasitol. 2007;116(4):340-5.

84. Gumushan-Aktas H, Altun S. Effects of Hedera helix L. extracts on rat prostate cancer cell proliferation and motility. Oncol Lett. 2016;12(4):2985-91.

85. Song J, Yeo SG, Hong EH, Lee BR, Kim JW, Kim J, Jeong H, Kwon Y, Kim H, Lee S, Park JH, Ko HJ. Antiviral activity of Hederasaponin B from Hedera helix against Enterovirus 71 subgenotypes C3 and C4a. Biomol Ther (Seoul). 2014; 22(1):41-6.

86. Hong EH, Song JH, Shim A, Lee BR, Kwon BE, Song HH, Kim YJ, Chang SY, Jeong HG, Kim JG, Seo SU, Kim H, Kwon Y, Ko HJ. Coadministration of Hedera helix L. Extract enabled mice to overcome insufficient protection against influenza A/PR/8 virus infection under suboptimal treatment with Oseltamivir. PLoS One. 2015;10(6):e0131089.

87. Majester-Savornin B, Elias R, Diaz-Lanza AM, Balansard G, Gasquet M, Delmas F. Saponins of the ivy plant, Hedera helix, and their leishmanicidic activity. Planta Med. 1991;57(3):260-2.

88. Al-Obaid $\mathrm{SOH}$. Studies on antibacterial and anticancer activity of Nerium oleander extracts. Eur Chem Bull. 2014;3(3):259-62.

89. Saleem F, Khan MTJ, Saleem H, Azeem M, Ahmed S, Shahid N, Gill MSA, Nadeem F, Ali T, Altaf H, Mehmood W. Phytochemical, antimicrobial and antioxidant activities of Pteris cretica I. (pteridaceae) extracts. Acta Pol Pharm. 2016;73(5):1397-403

90. Giliani AU, Ghayur MN, Khalid A, Zaheer ul H, Choudhary MI, Atta-Ur R. Presence of antispasmodic, antidiarrheal, antisecretory, calcium antagonist and acetylcholinesterase inhibitory steroidal alkaloids in Sarcococca saligna. Planta Med. 2005;71(2):120-5.

91. Ghayur MN, Gilani AH. Studies on cardio-suppressant, vasodilator and tracheal relaxant effects of Sarcococca saligna. Arch Pharm Res. 2006;29(11):990-7.

92. McCutcheon AR, Roberts TE, Gibbons E, Ellis SM, Babiuk LA, Hancock RE, Towers GH. Antiviral screening of British Columbian medicinal plants. J Ethnopharmacol. 1995:49(2):101-10.

93. Rajbhandari M, Mentel R, Jha PK, Chaudhary RP, Bhattarai S, Gewali MB, Karmacharya N, Hipper M, Lindequist U. Antiviral activity of some plants used in Nepalese traditional medicine. Evid Based Complement Alternat Med. 2009;6(4):517-22.

94. Escobar FM, Sabini MC, Zanon SM, Tonn CE, Sabini LI. Antiviral effect and mode of action of methanolic extract of Verbascum thapsus $L$. on pseudorabies virus (strain RC/79). Nat Prod Res. 2012;26(17):1621-5.

95. Kashan ZF, Arbabi M, Delavari M, Hooshyar H, Taghizadeh M, Joneydy Z. Effect of Verbascum thapsus ethanol extract on induction of apoptosis in Trichomonas vaginalis in vitro. Infect Disord Drug Targets. 2015;15(2):125-30.

96. Aktay G, Deliorman D, Ergun E, Ergun F, Yeşilada E, Çevik C. Hepatoprotective effects of Turkish folk remedies on experimental liver injury. J Ethnopharmacol. 2000;73(1):121-9.

97. Kiran K, Saleem F, Awan S, Ahmad S, Ahmad S, Malik MAA, Akhtar B, Raza M, Peerzada S, Sharif A. Anti-inflammatory and anticancer activity of Pteris cretica whole plant extracts. Pak Vet J. 2018;38(3):225-30.

98. Kant TH, Zabeer A, Pooja Samanta KC. Antihyperglycemic activity of petroleum ether extract \& ethyl acetate extract of Sarcococca saligna. Int J Curr Pharm Res. 2011;3(1):26-9.

99. Lans C, Turner N, Khan T. Medicinal plant treatments for fleas and ear problems of cats and dogs in British Columbia, Canada. Parasitol Res. 2008; 103(4):889-98

100. Khan MF, Tang H, Lyles JT, Pineau R, Mashwani Z-u-R, Quave CL. Antibacterial properties of medicinal plants from Pakistan against multidrugresistant ESKAPE pathogens. Front Pharmacol. 2018;9(815). https://doi.org/ 10.3389/fphar.2018.00815.

\section{Publisher's Note}

Springer Nature remains neutral with regard to jurisdictional claims in published maps and institutional affiliations. 